\title{
Comparison of routing methods in telecommunication networks - an overview and a new proposal using a multi- criteria approach dealing with imprecise information
}

\author{
João Clímaco ${ }^{1}$, José Craveirinha ${ }^{1}$, Lúcia Martins ${ }^{1,2}$ \\ ${ }^{1}$ Institute for Systems Engineering and Computers at Coimbra, INESC-Coimbra, University \\ of Coimbra, Portugal.e-mail: jclimaco@fe.uc.pt \\ ${ }^{2}$ Dept. of Electrical Engineering and Computers - Faculty of Sciences and Technology of \\ the University of Coimbra, Portugal
}

\begin{abstract}
The performance evaluation and comparison of routing models in telecommunication networks, normally imply the necessity of evaluating them through multidimensional, potentially conflicting, often incommensurate criteria, frequently involving imprecise information regarding the relative importance of the various network performance criteria. As we will show, this is particularly relevant for flow-oriented, decentralized routing optimization methods, having in mind their inherent limitations. Therefore, we formulate a decision problem focused on the comparison and selection of flow-oriented routing models, evaluated through multiple global network performance measures. A proposal of a multi-criteria/multi-attribute approach for tackling this decision problem, based on the VIP (Variable Interdependent Parameter) software, will be described. The adequacy of the features of the multi-attribute decision analysis model, which uses additive aggregation of criteria with variable interdependent importance parameters, coping with imprecise information, will be discussed. A detailed formulation of the application of the proposed approach to a specific problem involving the choice of a point-to-point routing method in a modern transport telecom network, from a set of height routing models, by considering their performance evaluated in terms of nine global network performance measures, will be presented. Moreover, the extension of the decision analysis model, based on the VIP decision support tool, for dealing with this problem, in the case of face-to-face cooperative group decision, will be addressed. A case study concerning the application of this approach, to the aforementioned decision problem, in a setting involving three decision makers, including a facilitator, will be presented. Finally, some conclusions, both from a methodological and practical nature, founded on the application study, will be put forward, highlighting the interest of this type of approach in this important area of telecom-network design.
\end{abstract}

Keywords: multi-criteria/multi-attribute decision analysis; telecommunication networks, routing methods 


\section{Introduction and Motivation}

\subsection{Introduction and Background Concepts}

In the general context of network design activities, routing is a fundamental issue that may be envisaged as part of the network operational planning decision process, strongly related to other network design steps, namely network structure design (which includes topological design and capacity facility dimensioning) and traffic network management. Routing methods may have different natures and a great multiplicity of formulations, depending fundamentally on the following factors: mode of information transfer and possibly other key technological features, the type of service(s) associated with the routed traffic flows, the level(s) of representation of the network, the features of the routing principle, associated with the objective(s) (to be optimized), the constraints to be satisfied, the time dependence of the execution of the routing algorithm and the information for the routing calculation. Routing methods are, in practice, technically implemented, in a given network environment, involving multiple technical factors, trough 'routing protocols'. Concerning the levels of network representation, for example, at least two levels are considered when resilience objectives are considered in the network design: the physical or transmission network and the logical or functional network. The physical network includes the transmission systems (such as optical fiber cables or microwave links and associated transmitter/receiver equipment), the switching and/or routing devices and their physical interconnections. The logical network is an upper level simplified representation of the network, of mathematical nature, over which the routing functionalities can be specified through logical rules and includes, as basic elements, a capacitated graph and a matrix of node to node offered demand, where the nodes typically represent switches or routers and the arcs transmission capabilities between the end nodes. As for the concept of 'routing principle' we mean the fundamental features of the routing method, for example whether it is concerned with node-to-node or with node-tomultiple nodes connections, or whether it is static or dynamic, i.e. if routes are time varying according to traffic fluctuations or network conditions, in a given time scale.

The extremely fast pace of the evolution in basic network technologies and architectures has led, in recent years, to a sharp increase in the proposals of routing models for different types of networks. Note that different routing methods often lead to different routing solutions for each node-to-node flow or VC (Virtual Connection, i.e. a specific node-to-node logical connection, with a given bandwidth requirement) in a given network environment, for given traffic offered to the network, solutions which are specified by a sequence of network resources, topologically defined by loopless paths in the network representation, in the case of point-to-point communications. Similar comments apply to point-multipoint or multipoint-multipoint communications, in which case Steiner trees or spanning trees have to be calculated in the network representation, according to the nature of the communication service. Reference monographs on routing models for telecommunication networks, including key mathematical formulations and algorithms can be seen in [Pióro \& Medhi 2004] and [Medhi \& Ramasamy 2018]. 
Most routing models, proposed in recent years, may be included in the category of 'QoS (Quality of Service) routing models', a type of routing that involves the selection of a chain of network resources along a feasible path explicitly satisfying certain requirements (dependent on traffic features associated with service types) and seeking to optimize some relevant metric such as delay, cost, number of edges of a path or loss probability. Therefore, in this context, routing algorithms need to consider as objective function or constraint(s), distinct path metrics. Comprehensive reviews on classical constrained-based QoS routing models and algorithms are provided in [Kuipers et al. (2002a), Kuipers et al. (2002b)]. In various modern network design situations there are advantages in formulating routing problems, as explicit multi-criteria optimization problems, where two or more metrics or cost functions are considered as objective functions to be optimized. Note that this type of formulations enable the trade-offs among distinct path metrics and other network cost function(s), to be pursued in a mathematically consistent manner [Clímaco et al. 2007]. An overview on multi-criteria routing models in telecommunication networks including the discussion of a case study can be seen in [Clímaco et al. 2007]. A recent state of art review on applications of multi-criteria analysis in telecommunication network planning and design problems, including a section on multi-criteria routing models is in [Clímaco et al. 2016].

For scalability considerations, most proposals of routing methods, from classical single-criterion optimisation models and QoS routing methods (based on multiple variants of constrained shortest path exact algorithms or heuristics) to explicitly multicriteria routing optimization models, are decentralised flow-oriented routing models. Alongside other authors (see, for example [Mitra et al. 1999]) we make the important distinction (see the analysis in [Craveirinha et. al. 2008]), between flow-oriented routing optimization models, for which routing calculation is performed for each node-to-node flow separately, in terms of a path optimization problem (with one or multiple objective functions and constraints), and network-wide optimization routing models. That is, flow oriented optimization routing approaches are characterized by objective function(s) which are defined on a per connection demand basis, i.e. for each offered end to end traffic flow - this means that the routing optimization model is solved separately every time a new end to end traffic flow is considered in the network. In contrast, network-wide optimization routing approaches consider the objective function(s) defined at network level, i.e. specified as global network performance measures that depend, explicitly and simultaneously, on all traffic flows present in the network, this implying that the optimization model is solved considering explicitly the combined effect of all network flows in all links/edges of the network - for example, through global network flow programming formulations.

As noted in [Craveirinha et. al. 2008], in flow oriented optimization routing approaches the objective functions, although closely related to the global network performance measures which we are "really" seeking to optimize, are unable to encompass consistently all the interactions among all network flows, the reason why the specified objective functions for any end-to-end flow can be designated as "surrogate" objective functions with respect to the ones in the associated network-wide optimization routing models. For example, the optimization of a given path metric (such as mean delay, blocking probability or load cost) for an end-to-end flow, seeks to optimize the corresponding global network metric. This is an inherent limitation of flow 
oriented optimization routing models in comparison with network-wide optimization routing models, which often leads to routing solutions that are poor in terms of the global network performance metrics.

For these reasons it is necessary to evaluate the performance of flow oriented routing models through relevant global network performance measures, to be specified by the network designer in a given network environment. Furthermore, QoS and multicriteria routing models should be compared, among themselves and with more conventional single-criterion routing optimisation models, by considering various relevant network performance measures. In the vast literature on routing methods, their performance, including performance comparisons among routing methods in a given network context, has typically been carried out through experimental studies involving simulation of multiple network scenarios followed by an empirical comparison of the network performance results obtained with the compared routing solutions.

A few examples of typical performance comparisons studies on flow-oriented routing methods, in various types of network environments, may now be referred to, in order to illustrate the analysis above. In [Meghanathan et al. 2009] a performance comparison study of three different types of routing protocols for mobile wireless ad hoc networks (or "MANETS"), is presented. A simulation test-bed is used and these routing methods are compared and ranked in terms of average packet delivery ratio, number of route transitions, average hop count and end-to-end packet delay, considering these criteria separately, by using multiple graphics for these metrics, in different conditions. The paper [Iver et al. 2013] presents a performance comparison of three routing protocols for wireless "smart utility networks", a specific type of wireless adhoc networks. The heuristic routing methods implemented by such protocols are compared, using a discrete event stochastic simulation, in terms of transmission resource usage (measured by the hop-count), average packet delay and average packet delivery ratio, considering three types of services and also taking into account route reliability. The performance criteria are directly compared through graphical representations. The work [Sllame et al. 2015] presents a performance comparison of a Voice over Internet Protocol (VoIP) communication service working over wireless ad hoc networks, considering multiple alternatives defined in terms of three different routing protocols and three different queuing techniques. The performance comparison uses four technical criteria, concerning jitter, packet delays and packet delivery ratio. Multiple graphics, obtained by real-time simulation, are used for empirical comparison of the alternatives, where, for each routing protocol, the criteria values are displayed, considering the various packet queuing techniques that may be used at the nodes.

The results of these, and of similar studies involving the comparison of routing methods, are typically expressed through statements of the type: "The method A has better performance (or a certain \% improvement in performance) than method B with respect to network metric $X$, in load condition $L, \ldots$. . In 'robust' and more technically sound studies, multiple network performance metrics/costs should be considered for comparison purposes, also having in mind that most flow-oriented routing models and also, possibly, network-wide optimisation routing models unable of encompassing explicitly some relevant network performance metrics - tend to perform asymmetrically with respect to different network metrics. This makes that the comparison and 
ultimately the choice - by a network design expert, working for a given network operator- of "a routing method with better overall performance" in a given network context, may easily become a difficult task, involving what is, in fact, a complex decision problem.

\subsection{Motivation and Contents}

As explained above, the inherent limitations of flow-oriented optimization routing models, make that the global effect of the interactions between traffic flows is not fully represented in their objective functions, justifying why we focused our study on the application of multi-attribute decision analysis to the comparison and selection of routing models of this type. In fact, these routing models may treat in quite an unbalanced manner, various relevant network performance metrics and may lead to poor performance in some of those metrics. The interest and necessity, in our view, of developing a decision support model, with sound methodological foundations, for tackling the mentioned type of evaluation and decision problem, of great practical interest in this specific area of network design, laid a major motivation for this work.

The decision problem at stake, is, from an Operational Research point of view, a problem involving the ranking of decision alternatives according to multiple criteria/attributes, where the alternatives are in a small number, are known explicitly, and the attributes correspond to the network global performance parameters chosen for evaluation purposes, often conflicting and incommensurate.

A major contribution of our study is to show the usefulness and potential, both from a methodological and practical point of view, of using a certain multi-attribute (MA) analysis model dealing with incomplete information. A reference monograph on key concepts concerning multi-attribute models can be seen in [Keeney \& Raiffa 1979].

The used MA model will consider an additive value function (see [Keeney \& Raiffa 1979]) which is constructed through a weighted sum of the attribute values. Remember that the attribute values have to be previously normalized. Furthermore, at each evaluation exercise, the fixing of the values of the scaling constants/weights, corresponding to criteria importance parameters assigned to the various attributes, has to be tackled. Note that this is a most difficult task, since the relative importance, particularly in quantitative terms, among the different attributes, is normally imprecise. For example, the question "how more important is mean total carried traffic than total residual bandwidth?" may have a variety of answers in terms of network routing design. That is why the imprecise nature of the scaling constants is a fundamental feature of the addressed decision problem, that has an important role in the specification of the MA analysis model. Moreover, note that various attributes may be conflicting and incommensurate, as the two exemplified above (mean total carried traffic and total residual bandwidth).

Furthermore, the interest in considering a group decision process in this application environment has to do with several aspects. Firstly, although some general assumptions on relative importance of the network performance metrics may be common to most network designers, the specification of relations between the criteria IPs (im- 
portance parameters) may vary significantly from one expert to another, even when those differences of perspective, in terms of systems of preferences, are not assumed explicitly, but just tacitly. This is also reflected in the literature in this area where some authors give more relevance to certain performance measure than others, when analyzing and comparing the performance of routing methods in a given network environment (see examples, in the overview of routing models in [Craveirinha et al. 2008] and [Clímaco et al. 2016]). The need for the elicitation of such differences and the analysis of their consequences in the network design process, is one of the advantages of considering a setting of group decision in the context of this particular multi-criteria decision problem. Secondly, this may be a realistic decision scenario in the context of a major network operator, since more than one engineer/network designer is often involved in a decision which has decisive impact in terms of network performance, cost and expected revenues. This can be a typical situation when new, more advanced, routing methods are to be implemented in a transport network, for example provided by the development by the operator or by a company of a related group, of more sophisticated routers, in relation to the deployment of modern telecommunication protocol technologies, for example the MPLS-TP (Multiprotocol Label Switching-Transport Profile) stack [Niven-Jenkins et al. 2009]. The authors themselves were involved in research contracts of collaboration of their research institute (INESC-Coimbra) with Portugal Telecom-Innovation, where this type of decision environments and issues were at stake.

In this study, we consider, as alternatives of the formulated decision problem, several variants of flow-oriented routing models, namely, in the presented case study, different variants of a bi-criteria flow oriented routing model, as well the two associated single-criterion routing models. Hereafter, we designate as bi-criteria routing model, a routing model characterized by two specific objective functions. We consider, in our case study, two different forms of normalizing the objective functions and two forms of aggregation of preferences, leading to multiple variants of the routing model. Each of these variants corresponds to a routing method, in the sense defined in [Clímaco \& Craveirinha 2005]. In the considered routing methods, for technical requirements, the aggregation of preferences is performed automatically, as explained later on. The attributes of the decision problem are various network performance metrics that enable the evaluation of the global effect, at network level, of using the various routing methods, when incremental traffic is offered to a given transport network. These performance metric values were obtained in the context of a previous study [Martins et. al. 2013] on network performance improvement through evaluation of bi-criteria/single-criterion flow oriented routing methods in transport networks, focused on applications in Carrier-Ethernet [MEF 10.3, 2013] and MPLS-TP networks. This study was focused on bandwidth allocation and traffic performance, having in mind its paramount importance in packet transport networks, in relation with the design of adequate routing methods, capable of obtaining improved network performance and prevent degradation of the QoE (Quality of Experience) as perceived by the users, specially in overload conditions.

As analyzed above, the imprecise information feature of the proposed MA model stems from the fact that the scaling constants, associated with the considered attributes are not fixed a priori, although various constraints between them can be set a priori as agreed among possible decision makers, for a given network operator. Of 
course, this is more realistic and flexible than requiring an a priori fixation of the scaling constants. Furthermore, although some of those inequality relations are consensual, for technical reasons, different decision agents, may assign different relative importance to the scaling constants associated with some attributes, or even consider different inequalities among them. Congruently, we will consider a multi-attribute analysis tool [Dias \& Clímaco 2000], the VIP Analysis package, which will enable the achievement of a compatibility of the incomplete information supplied by different scenarios of relative importance among the attributes (network performance metrics). The aim is that, as a final result of an interactive analysis process, some robust conclusions may be achieved, hence helping a well founded evaluation and choice of a routing method alternative, to be implemented in a particular network environment.

The main contributions of our work are the following:

- $\quad$ specification of a multi-attribute (MA) analysis model, based on the VIP analysis software, for comparing and selecting routing methods, in terms of multiple network performance measures, enabling the consideration by the network designer (decision maker) of multiple scenarios concerning different forms of valuation of the relative importance of the network performance measures, i.e. in a context of imprecise information on the importance parameters assigned by the DM to the network performance measures; this includes the extension of the decision analysis model, based on the VIP software, for face-to-face cooperative group decision;

- development of a case study of application of the (MA) analysis model to the comparison and selection of flow-oriented routing methods, namely methods based on bi-criterion shortest paths and single-criterion shortest paths algorithms, using as path metrics load costs and hop count, in the context of transport networks with incremental traffic; this application study, highlighting the capabilities of the used MA model, involves experiments with one DM and with three DMs, considering a realistic cooperative group decision setting.

- outline of relevant conclusions, of methodological and practical nature, founded on the MA analysis case study, concerning the relative performance of those types of flow-oriented routing methods, in various decision scenarios and putting in evidence the interest of this type of approach in this important area of telecommunication network design.

The contents of the paper are as follows. The next section describes the decision problem concerning the selection of flow-oriented routing methods, in a given transport network, and specifies the features of the multi-attribute analysis model, based on the VIP analysis package, used for tackling this problem. Section 3 presents the case study of application of the (MA) analysis model to the comparison and selection of flow-oriented routing methods, in the context of transport networks with incremental traffic, taking as inputs (attribute values) nine network performance metrics, the values of which were obtained from discrete event simulations. Multiple scenarios for the imprecise information on the importance parameters, as possibly assigned by the decision maker(s), were considered. Also some relevant conclusions, founded on the MA analysis case study, will be outlined in the second part of this 
section. Finally, section 4 presents the conclusions of this study and outlines further work on this this research theme.

\section{Outline of the Decision Problem and of the Multi-attribute Analysis Model}

\subsection{The Decision Problem}

\section{The alternatives}

The first six alternatives $\mathrm{a}_{\mathrm{i}}(\mathrm{i}=1, \ldots, 6)$ or solutions of the proposed decision problem, are variants of a bi-criteria flow-oriented routing model, in a transport telecommunication network considering incremental traffic, all using as path metrics, to be optimized, the load cost and the number of arcs (or "hop count"), the features of which are briefly reviewed next and in Appendix A. The other two alternatives are the two single criterion routing models that use, as path metric to be minimized, either the load cost or the hop count.

In order to briefly review these routing alternatives, let us consider a directed capacitated network $(\mathrm{N}, \mathrm{L}, \mathrm{C})$ where $\mathrm{N}$ is the node set, $\mathrm{L}$ the arc set and $\mathrm{C}$ the set of the capacities (total bandwidths) $\mathrm{C}_{\mathrm{k}}$ of the $\operatorname{arcs} \mathrm{l}_{\mathrm{k}}(\mathrm{k}=1, \ldots / \mathrm{L} /)$. Let $\mathrm{f}_{\mathrm{s}}$ denote a node to node traffic flow from node $v_{i}$ to node $v_{j}$ of service type 's'. A flow $f_{s}$ is associated with a virtual connection request (VC) requiring a certain bandwidth, $\mathrm{d}_{\mathrm{s}}$, in the used arcs, and may use a feasible loopless path or route $r_{s}$ - i.e. a loopless path from $v_{i}$ to $v_{j}$ such that every arc in $r_{s}$ has, at the moment of the arrival of the $\mathrm{VC}$ request, an available bandwidth $b_{k} \geq d_{s}$. The current set of feasible routes for flow $f_{s}$ is designated as $D\left(f_{s}\right)$. Let $\mathrm{m}_{\mathrm{k}}{ }^{\mathrm{i}}$ designate an additive metric, corresponding to the path metric $\mathrm{m}^{\mathrm{i}}\left(\mathrm{r}_{\mathrm{s}}\right)$ for any route $r_{s},(i=1,2)$, associated with every $\operatorname{arc} l_{k}$.

Then, the general basic bi-criteria flow oriented routing optimization model is formulated as:

$$
\boldsymbol{m i n}_{\boldsymbol{r}_{s}} \boldsymbol{m}^{\boldsymbol{i}}\left(\boldsymbol{r}_{\boldsymbol{s}}\right)=\sum_{\boldsymbol{l}_{\boldsymbol{k}} \in \boldsymbol{r}_{\boldsymbol{s}}}\left(\boldsymbol{m}_{\boldsymbol{k}}^{\boldsymbol{i}}\right) \quad(i=1,2) \wedge r_{s} \in D\left(f_{s}\right)
$$

In the considered model, the first path metric, $\mathrm{m}^{1}$, is the load cost that is expressed through a piecewise linear function in terms of the relative bandwidth occupation in the arcs, based on the model in [Fortz \& Thorup 2002] thenceforth depending, for arc $l_{k}$, on $b_{k}$ and $C_{k}$ (see details on this piecewise linear function in [Martins et. al. 2013]). The minimization of $\mathrm{m}^{1}\left(\mathrm{r}_{\mathrm{s}}\right)$ seeks to obtain a balanced traffic load distribution in the network arcs (or 'links'), preventing the use of excessively loaded links, while less loaded links are available, hence favoring traffic carrying capability for future VCs. The second metric is the number of arcs of the path (or hop count), i.e. $\mathrm{m}_{\mathrm{k}}{ }^{2}=1$, and its minimization seeks to minimize the number of network resources used by any VC. The bi-criteria flow oriented routing optimization model seeks to obtain a compromise solution in terms of these two, often conflicting objective functions. The final compromise solution, to be settled for each VC, has to be chosen in the set of non- 
dominated (or Pareto optimal) solutions. A solution $r$ ' is said to dominate the solution $r$ iff $m^{1}\left(r^{\prime}\right) \leq m^{1}(r)$ and $m^{2}\left(r^{\prime}\right) \leq m^{2}(r)$ and at least one of inequalities is strict. A path $\mathrm{r}^{*}$ is said to be non-dominated (or Pareto optimal) iff there is no other feasible path which dominates $\mathrm{r}^{*}$. The non-dominated paths were obtained by calculating k-shortest paths and by using a non-dominance test on the calculated solutions, as proposed in [Clímaco \& Martins 1982]. Note that this procedure enables the exact calculation of all non-dominated solutions of the bi-criteria shortest path problem. The variants of this basic bi-criteria routing model are related to different ways of automated selection of a "good" trade-off solution to the bi-criteria routing model (1), in the nondominated solution set, for each offered flow $f_{s}$, and to different forms of normalizing the two path cost functions. The parameters involved in the selection of a route depend not only on the current flow being offered to the network but also on the global network states, so they vary dynamically as more flows are offered to the network, in a scenario of stochastic incremental traffic. Note that the final routing solution selected for each VC offered to the network, is calculated in an automated manner by the routing management system. As for the way in which this selection is carried out, two types of methods were considered. The first type uses priority regions in the objective function space, defined from preference thresholds for the two objective functions, namely required and acceptable values $\mathrm{m}_{\text {req }}^{\mathrm{i}}, \mathrm{m}_{\mathrm{ac}}^{\mathrm{i}}(\mathrm{i}=1,2)$. This leads to four priority regions, as specified in [Martins et. al. 2013], so that the first non-dominated solution found in the highest priority region, is the one selected. The second type of method chooses the non-dominated solution which minimizes, either a Chebychev or a Euclidian distance to the optimal ideal point $\left(\mathrm{op}^{1}, \mathrm{op}^{2}\right)$, in the objective function space. The second factor that influences the selected solution is the choice of the normalizing coefficients of the objective functions, as explained in the Appendix A. Further details on the traffic modelling and calculation aspects, can be seen in [Martins et. al. 2013].

The other two alternatives, $a_{7}, a_{8}$, of the decision problem are, naturally, the single criterion routing optimization methods that use, as path metric to be minimized, either $\mathrm{m}^{1}\left(\mathrm{r}_{\mathrm{s}}\right)$, the load cost, or $\mathrm{m}^{2}\left(\mathrm{r}_{\mathrm{s}}\right)$, the hop count. The alternatives (routing methods) of our decision problem, are summarized in Table 1.

\begin{tabular}{ccc}
\hline $\begin{array}{c}\text { alternative } \\
\text { designation }\end{array}$ & $\begin{array}{c}\text { routing optimization } \\
\text { approach }\end{array}$ & solution selection \\
\hline $\mathrm{a}_{1}$ & bi-criteria & normalization by WA and priority regions \\
$\mathrm{a}_{2}$ & bi-criteria & normalization by WB and priority regions \\
$\mathrm{a}_{3}$ & bi-criteria & normalization by WA and Euclidian Distance \\
$\mathrm{a}_{4}$ & bi-criteria & normalization by WA and Chebychev Distance \\
$\mathrm{a}_{5}$ & bi-criteria & normalization by WB and Euclidian Distance \\
$\mathrm{a}_{6}$ & bi-criteria & normalization by WB and Chebychev Distance \\
$\mathrm{a}_{7}$ & single-criterion: load cost & \\
$\mathrm{a}_{8}$ & single-criterion: hop count & \\
\hline
\end{tabular}

Table 1: alternatives of the decision problem-routing methods 


\section{The attributes}

Concerning the attributes of the decision model, these are global network performance metrics involving three fundamental types of metrics: mean total carried bandwidth (TCB), mean total residual bandwidth (TRB) and mean number of accepted node-to-node VCs (TAC). Each of these fundamental metrics is decomposed into three attributes corresponding to the associated performance values obtained while the blocking probability of a connection request remains in zero $(\mathrm{Br} 1=0 \%)$ or attains the thresholds of $\mathrm{Br} 2=5 \%$ or $\mathrm{Br} 2=10 \%$, leading to a total of nine attributes.

The values for these performance measures in the network case study were estimated through stochastic discrete event simulations, considering incremental traffic and using the method of batch means for sample mean and confidence interval estimation.

\subsection{The Multi-attribute VIP Analysis}

The multi-attribute analysis model for tackling the considered decision problem is based on a decision support tool, the VIP Analysis software [Dias \& Clímaco 2000], [Dias \& Clímaco 2005] having in mind the adequacy of its features to the to the nature of our decision problem. This adequacy results, in first place, from the fact that the DM (the network designer) is not able or does not wish to establish a priori precise values for the importance parameters, or scaling constants, associated with the attributes - this is the imprecise information feature of the decision model. Note that the DM not only may find very difficult (or questionable under technical-economic reasons) to fix precise values for the importance parameters, but also may wish to consider and test various scenarios for the relative values of those parameters. This will become very clear, in the explanation of the MA package features, as well as in the case study in section 3, specially taking in account the way in which the scaling constants can be treated, namely as variable interdependent parameters, enabling, at the same time, to draw well founded conclusions concerning the ranking of alternatives.

Hence, to help the DM in a process of finding a most preferred alternative, the MA model considers an additive value function under imprecise information:

$$
V\left(\mathrm{a}_{i}, k\right)=\sum_{j=1}^{n} k_{j} v_{j}\left(\mathrm{a}_{i}\right) .
$$

where $a_{i}$ and $v_{j}$ represent the $i^{\text {th }}$ alternative and the $j^{\text {th }}$ normalized global network performance measure, of one of the types described above, $\mathrm{k}_{\mathrm{j}}$ is the importance parame-

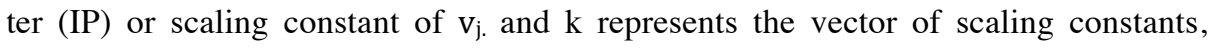
$\mathrm{k}=\left(\mathrm{k}_{1}, \mathrm{k}_{2}, \ldots, \mathrm{k}_{\mathrm{n}}\right)$.

Let us briefly review the most relevant concepts/definitions used in the multiattribute analysis package (see [Dias \& Clímaco 2000] for further details).

Let $\mathrm{T}$ denote the set of acceptable values of the vector $\mathrm{k}$ of scaling constants. 
The $\operatorname{regret}\left(\mathrm{a}_{\mathrm{i}}, \mathrm{a}_{\mathrm{j}}\right)$ associated with alternative $\mathrm{a}_{\mathrm{j}}$, when compared with $\mathrm{a}_{\mathrm{i}}$, and here denoted as $\mathrm{reg}_{i j}$, is the maximal difference:

$$
r e g_{i j}=\max _{k \in T}\left\{V\left(\mathrm{a}_{i}, k\right)-V\left(\mathrm{a}_{j}, k\right)\right\}
$$

If $\quad r e g_{i j}$ is negative then $V\left(\mathrm{a}_{j}, k\right) \geq V\left(\mathrm{a}_{i}, k\right) \forall k \epsilon T \quad \wedge \exists k \epsilon T: V\left(\mathrm{a}_{j}, k\right)>$ $V\left(\mathrm{a}_{i}, k\right)$ and $\mathrm{a}_{\mathrm{j}}$ is said to dominate $\mathrm{a}_{\mathrm{i}}$, (i.e. $\mathrm{a}_{\mathrm{i}}$ is dominated by $\mathrm{a}_{\mathrm{j}}$ ). An even more demanding situation of dominance, corresponds to the case:

$$
V\left(\mathrm{a}_{j}, k\right) \geq V\left(\mathrm{a}_{i}, k^{\prime}\right) \quad \forall \mathrm{k}, \mathrm{k}^{\prime} \in \mathrm{T} \wedge \exists \mathrm{k}, \mathrm{k}^{\prime} \in \mathrm{T}: V\left(\mathrm{a}_{j}, k\right)>V\left(\mathrm{a}_{i}, k^{\prime}\right)
$$

In this case we say that $\mathrm{a}_{\mathrm{i}}$ is absolutely dominated by $\mathrm{a}_{\mathrm{j}}$.

A relaxation to the dominance relation, by a tolerance parameter $\varepsilon$, means that:

$$
V\left(\mathrm{a}_{j}, k\right) \geq V\left(\mathrm{a}_{i}, k\right)-\varepsilon \forall k \epsilon T \wedge \exists k \epsilon T: V\left(\mathrm{a}_{j}, k\right)>V\left(\mathrm{a}_{i}, k\right)-\varepsilon
$$

In this case we say that $\mathrm{a}_{\mathrm{j}}$ quasi dominates $\mathrm{a}_{\mathrm{i}}$ with tolerance $\varepsilon$.

For every alternative $\mathrm{a}_{\mathrm{i}}$ the maximal regret associated with it, when it is compared with all other alternatives which may have a higher additive value for given $\mathrm{T}$, is:

$$
r e g_{\max }\left(\mathrm{a}_{i}\right)=\max _{j \neq i}\left\{r e g_{j i}\right\}=\max _{k \in T}\left\{\max _{j \neq i}\left\{V\left(\mathrm{a}_{j}, k\right)\right\}-V\left(\mathrm{a}_{i}, k\right)\right\} \text {. }
$$

If $\operatorname{reg}_{\max }\left(\mathrm{a}_{\mathrm{i}}\right)$ is negative or null then $\mathrm{a}_{\mathrm{i}}$ is optimal; if $\operatorname{reg}_{\max }\left(\mathrm{a}_{\mathrm{i}}\right)-\varepsilon$ is negative or null then $\mathrm{a}_{\mathrm{i}}$ is quasi-optimal. If this is true only for a subset $\mathrm{K}^{*}$ of $\mathrm{T}$ then we can say that $\mathrm{a}_{\mathrm{i}}$ is $k$-optimal (or quasi optimal) at $\mathrm{K}^{*}$.

VIP Analysis [Dias \& Clímaco 2000] is an interactive software package dedicated to the choice problematic regarding the evaluation of a discrete set of alternatives according to a multi-attribute additive value function. The principal characteristic of this tool is that no precise values, for the scaling constants, are required. Instead, it can accept imprecise information (typically, intervals and/or linear constraints) on these values, usually identified by indirect ways, as for example by comparing swings. The major objectives are the discovering of robust conclusions - that may be shown mathematically to hold for every feasible combination of the scaling constants - and, secondly, identifying what is the variability of the results due to the imprecision in the parameter values. Furthermore, by considering multiple specifications of the set $\mathrm{T}$, the DM may address the choice of a best compromise alternative, in different scenarios, that he/she finds more relevant on technical-economic grounds or more consistent with his/her experience as network designer.

In a first phase of the MA procedure some tools enable filtering the alternatives. In particular, the VIP module calculates the range of values of each alternative:

$$
\left[\min _{k \in T}\left\{V\left(\mathrm{a}_{i}, k\right)\right\}, \max _{k \in T}\left\{V\left(\mathrm{a}_{i}, k\right)\right\}\right] .
$$


This enables, for example, the elimination of the alternatives with a minimum value below a certain threshold (fixed by the decision makers) regarding: the maximum regret concerning each alternative (enabling the elimination of those with a max regret beyond a threshold fixed by the decision makers); possibly the elimination of absolutely dominated or dominated solutions. It must be remarked that the identification of the alternative(s) with "min-max regret" constitutes an indicator regarding equity and the identification of the alternative(s) with "max min value" is an indicator regarding "best case". The alternatives passing the filtering phase are analysed using the matrix of regrets, $\left[\right.$ reg $\left._{i j .}\right](\mathrm{i}, \mathrm{j}=1, \ldots \mathrm{m})$, defined from (3), designated as pairwise confrontation table. In conjunction with the relaxation of the concepts of optimality and dominance, this enables the exploration of the concepts of quasi-optimality and quasi-dominance, enabling the decision maker to identify robust conclusions, in order to help in the search for the best alternative. Also note that, in those cases for which the DM is able of fixing some trade-offs between pairs of criteria, it is possible to reduce the number of independent variable scaling constants. When just three independent variables remain, the system provides a graphical representation of the space of the scaling constants, allowing a user-friendly representation of the optimality and quasi-optimality domains.

In the addressed network design decision problem, a final routing method alternative must be chosen. In theory two situations may occur at the end of the analysis process: the most common, where one alternative becomes the one clearly preferred by the DM, in all admitted scenarios for $\mathrm{T}$, as a result of its inherent merits in a given network environment, or a situation in which the DM finds that at least two alternatives are worthy being considered, since neither of them is clearly preferred to, in the admitted scenarios. In this case the DM may have two courses of action: either to consider a 'narrowing' of the conditions which specify the set $\mathrm{T}$, leading, through a complementary process of analysis with the VIP package, trying to clarify the situation, or to present those best alternatives to a 'higher hierarchy' DM of the network operator, and confront him/her with the pros and cons of those alternatives, so that he/she makes an ultimate selection in this final short list of alternatives.

As for the upgrading of these concepts to Group VIP Analysis (see [Dias \& Clímaco 2005]), for instance, concerning the concept of "quasi-domination of alternatives", the DMs tolerance $\varepsilon$ may vary jointly with a " $\alpha$-majority operator". In the case study dealt with in this paper, we consider three decision makers, so $\alpha \in\{1 / 3,2 / 3,1\}$. In fact, the DM's may be interested in analyzing which alternatives are quasi-dominated, in terms of an " $\alpha$-majority rule". Note that the obtainment of a quasi-dominance condition with a high value of $\alpha$ may imply a high value for $\varepsilon$ - an example is shown in the case study described in the next section. Of course this type of approach can also be used for other types of decision issues. 


\section{Case Study}

\subsection{Main Features of the MA Application Model}

A reference network based on the France telecommunication transport network, described in [Martins et. al. 2013] and with topology given in [Orlowski et al. "SNDLIB" 2010], considering that all links (arcs of the network topology) have 10 $\mathrm{Gb} / \mathrm{s}$ capacity and three connection service types, was considered. The three service types were assumed to have effective bandwidths of 20,50 and 100Mbit/s. In the stochastic simulation study, point to point $\mathrm{VC}$ requests were randomly generated over all origin-destination pairs and service types, considering uniform distributions. The accepted VCs are assumed to be maintained 'indefinitely' in the network (that is the established routes correspond to physical paths which are held during the routing study time scale), which corresponds to the usual assumption of offered traffic of incremental type, typical of routing studies in transport networks. The study in [Martins et. al. 2013], was carried out in collaboration with experts of Portugal Telecom Inovação and was focused on the evaluation of the bandwidth allocation and traffic performance when various point to point routing methods, as the ones described in Table 1, were applied in the context of Carrier-Ethernet and MPLS-TP (Multiprotocol Label Switching-Transport Profile) transport networks. The estimated values for the network performance metrics, used as attributes in the present MA model, were obtained by considering 100 simulation replicas of incremental traffic and their average values were calculated, from raw data collected during the experiments in [Martins et. al. 2013].

As mentioned above, a total of nine attributes were considered. The corresponding values were normalized by using difference ratios, i.e. the estimate $\mathrm{y}_{\mathrm{j}}\left(\mathrm{a}_{\mathrm{i}}\right)$ of the $\mathrm{j}^{\text {th }}$ network performance metric, for a given alternative $a_{i}$, is normalized into the attribute value in $[0,1]$ :

$$
v_{j}\left(\mathrm{a}_{i}\right)=\frac{y_{j}\left(\mathrm{a}_{i}\right)-y_{j \min }}{y_{j \max }-y_{j \min }} ; y_{j \min }=\min _{\mathrm{a}_{k}}\left\{y_{j}\left(\mathrm{a}_{k}\right)\right\}, y_{j \max }=\max _{\mathrm{a}_{k}}\left\{y_{j}\left(\mathrm{a}_{k}\right)\right\}
$$

The first three attributes (or criteria) $\mathrm{v}_{\mathrm{j}}(\mathrm{j}=1,2,3)$, are the mean total carried bandwidths (TCB) while the blocking probability of a $\mathrm{VC}$ request remains in zero $(\mathrm{Br} 1=0 \%)$ or attains the thresholds of $\mathrm{Br} 2=5 \%$ or $\mathrm{Br} 2=10 \%$, respectively, and the corresponding scaling constants are denoted by $k_{j}=k_{(j) T C B} \mathrm{j}=(1,2,3)$. The attributes $\mathrm{v}_{\mathrm{j}}(\mathrm{j}=4,5,6)$ are the mean total residual bandwidths (TRB) defined in the same conditions as above, and the corresponding scaling constants are denoted by $k_{j+3}=k_{(j) T R B} \mathrm{j}=(1,2,3)$. Finally the attributes $\mathrm{v}_{\mathrm{j}}(\mathrm{j}=7,8,9)$ are the mean numbers of accepted node-to-node VCs (TAC), defined in the same conditions, and the corresponding scaling constants are denoted by $k_{j+6}=k_{(j) T A C} \mathrm{j}=(1,2,3)$. 


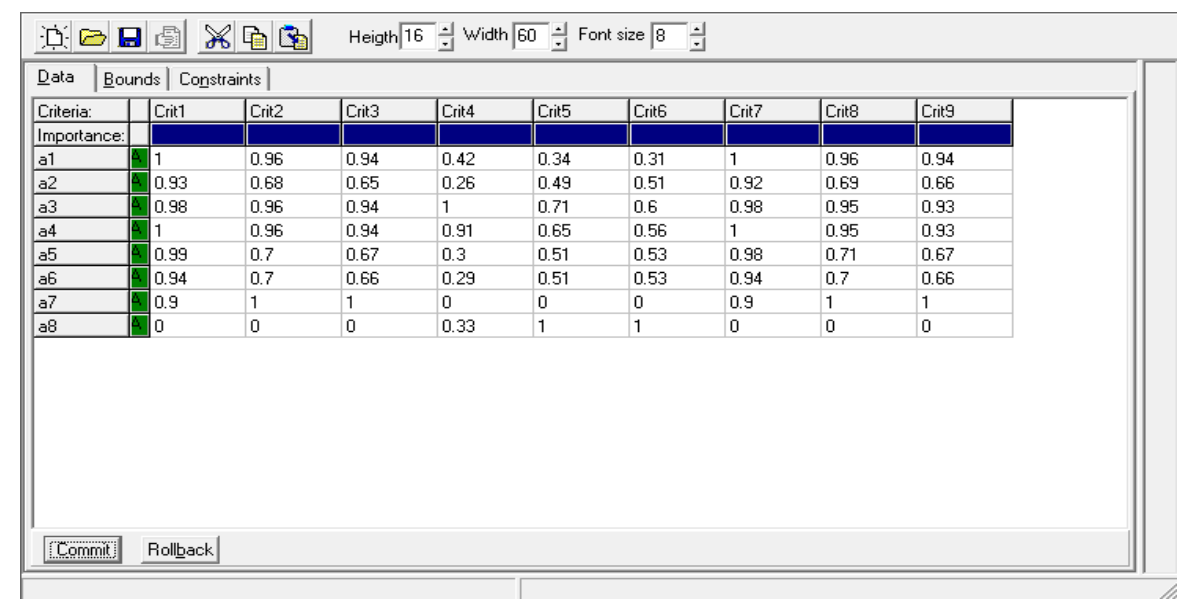

Fig.1- Normalized performance matrix

The obtained values $\mathrm{v}_{\mathrm{j}}\left(\mathrm{a}_{\mathrm{i}}\right)$ are shown in the form of the normalized performance table of the VIP package, in Fig.1.

\subsection{Experimental Study with a single decision maker}

We begin by presenting two sets of experiments involving two separate decision makers, DM1 and DM2, whose constraints on the IPs (importance parameters) reflect common assumptions of network designers regarding the relative importance of the performance metrics. This will enable the potentialities of the proposed MA analysis model to be illustrated, in our decision environment.

\section{First set of experiments (DM1)}

The first set of experiments, regarding the first decision maker, DM1, was carried out considering a total of 15 constraints on the scaling constants, which are either inequality relations (corresponding to 13 constraints) or equality relations (2 constraints). These constraints correspond to the general assumptions of most network designers, making it explicit the relative importance of some pairs of network metrics, when evaluating routing methods, namely considering that: i) the total carried bandwidth (TCB) and the total number of accepted connections (TAC) measures are more relevant than total residual bandwidth (TRB) measures, for the same level of blocking probability; ii) for a given type of network metric (namely associated with TCB or TAC) the measure for blocking probability $\mathrm{Brl}(0 \%)$ is more important than the measure for $\mathrm{Br} 2(5 \%)$ and similarly for measures for $\mathrm{Br} 2$ and $\mathrm{Br} 3(10 \%)$, excepting in the case of the measures for TRB, a situation in which those preferences are the reverse. The equality relations concern the measures TCB and TAC, for Br1 and Br2. In this first scenario of relations between scaling constants it is further assumed that the 
performance in terms of TCB is at least as important than TAC for blocking probability $\mathrm{Br} 3(10 \%)$. This set of constraints (denoted as constraint set scenario S1) is expressed, according to the defined notation:

$$
\begin{array}{rlrl}
k_{(j) T C B} & >k_{(j+1) T C B}(j=1,2) & & (c 1) \\
k_{(j) T R B} \prec k_{(j+1) T R B}(j=1,2) & & (c 2) \\
k_{(j) T A C} & >k_{(j+1) T A C}(j=1,2) & & (c 3) \\
k_{(j) T C B} & >k_{(j) T R B}(j=1,2,3) & & (c 4) \text { Constraint set S1 } \\
k_{(j) T A C} & >k_{(j) T R B}(j=1,2,3) & & (c 5) \\
k_{(j) T C B} & \equiv k_{(j) T A C}(j=1,2) & & (c 6) \\
k_{(j) T C B} & \geqslant k_{(j) T A C}(j=3) & & (c 7)
\end{array}
$$

Here, " $>$ " denotes "more important than", " $\geqslant$ " means "at least as important as" and " $\equiv "$ denotes "as important as". Note that the VIP software uses the simplex method, therefore strict inequality relations cannot be used, so that we have to use, to implement such relations, a small perturbation of " $\geq$ " or " $\leq$ " relations.

The summary of the main features of the alternatives, in this experiment, is shown in fig. 2, where the corresponding VIP table is reproduced as actually seen in the computer screen. The left hand side of this picture is just a part of the table, used in VIP software, to introduce the constraints S1, the remainder of that table is not shown since it does not add any significant information. This first experiment enabled to identify one alternative absolutely dominated $\left(a_{8}\right)$, i.e. its more favorable value is lower than the worst value of other alternative(s), and with very poor relative values in almost all attributes, and four alternatives which were dominated by $a_{3}$ or by $a_{4}$. Moreover, $a_{3}$ was the solution with maximal minimal value and $a_{4}$ the solution with minimal maximal regret. The pairwise confrontation table is shown in fig. 3 and graphics with the min-max ranges and the maximal regrets for the eight alternatives, are shown in figs. 4 and 5, respectively, enabling a visual assessment of the relative performances of the alternatives concerning these important features of the MA model results.

In this set of experiments various runs of the VIP software were carried out varying the level of dominance relation relaxation, i.e. quasi-dominance, specified by the relative tolerance $\varepsilon$ (different $\varepsilon$ values were considered for testing quasi-dominance or "reinforced" dominance - negative $\varepsilon$ ). This was done by sliding in a horizontal screen bar, provided by the software.

The consideration of a small relaxation to dominance revealed, by examining the confrontation table, that all alternatives (different from $\mathrm{a}_{3}, \mathrm{a}_{4}$ ) were quasi-dominated by $\mathrm{a}_{3}$ or $\mathrm{a}_{4}$, for $\varepsilon \geq 0.01$, as illustrated in the summary of fig 6 . and through the confrontation table in fig 7 , obtained for $\varepsilon=0.01$. 


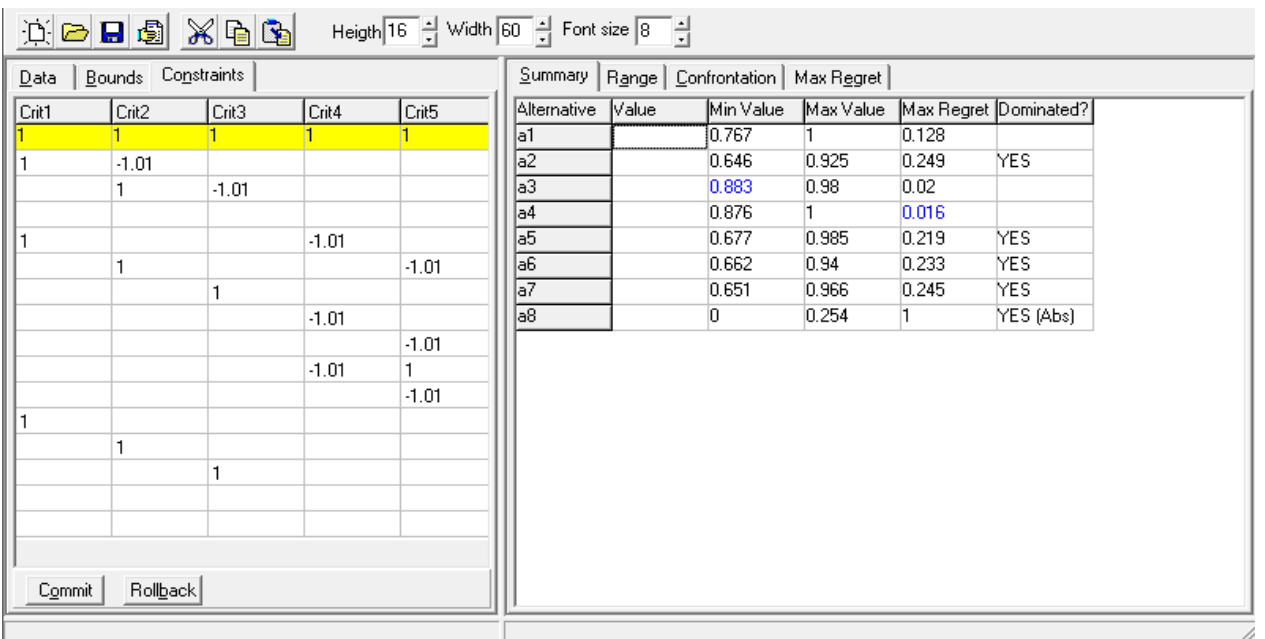

Fig.2 Main features of alternatives, for S1.

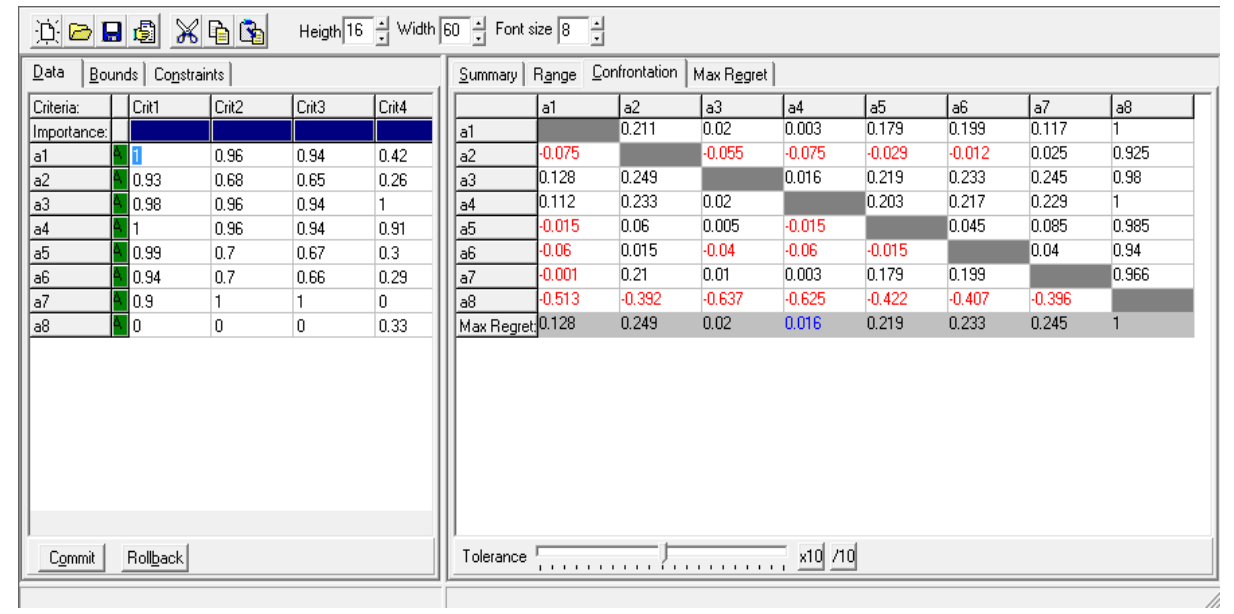

Fig.3 The pairwise confrontation table, for S1. 


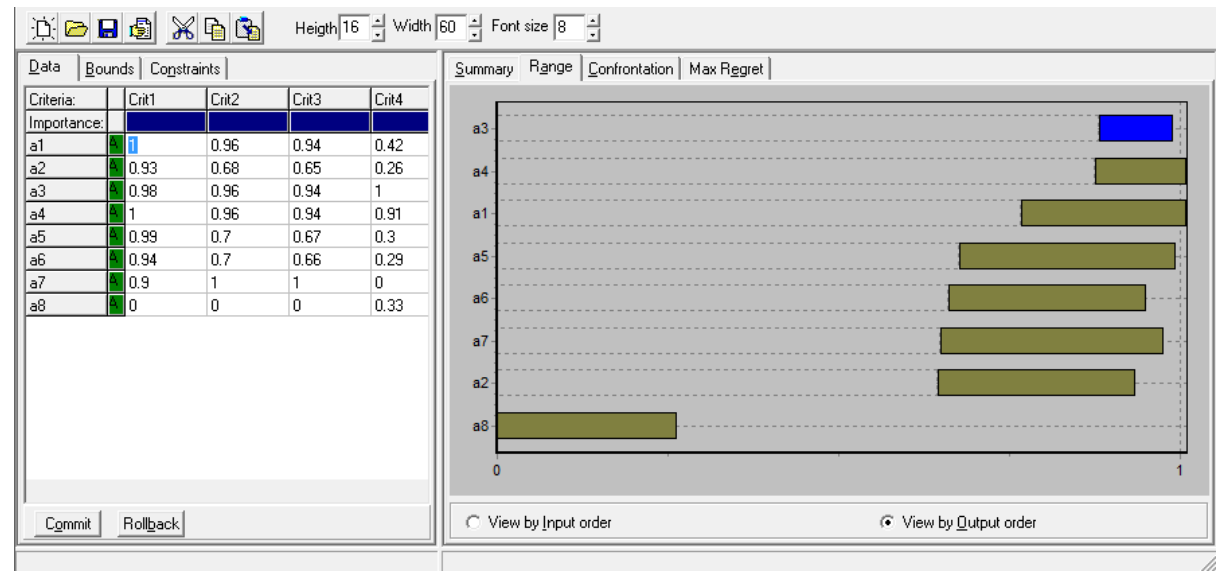

Fig. 4 The min-max ranges for S1.

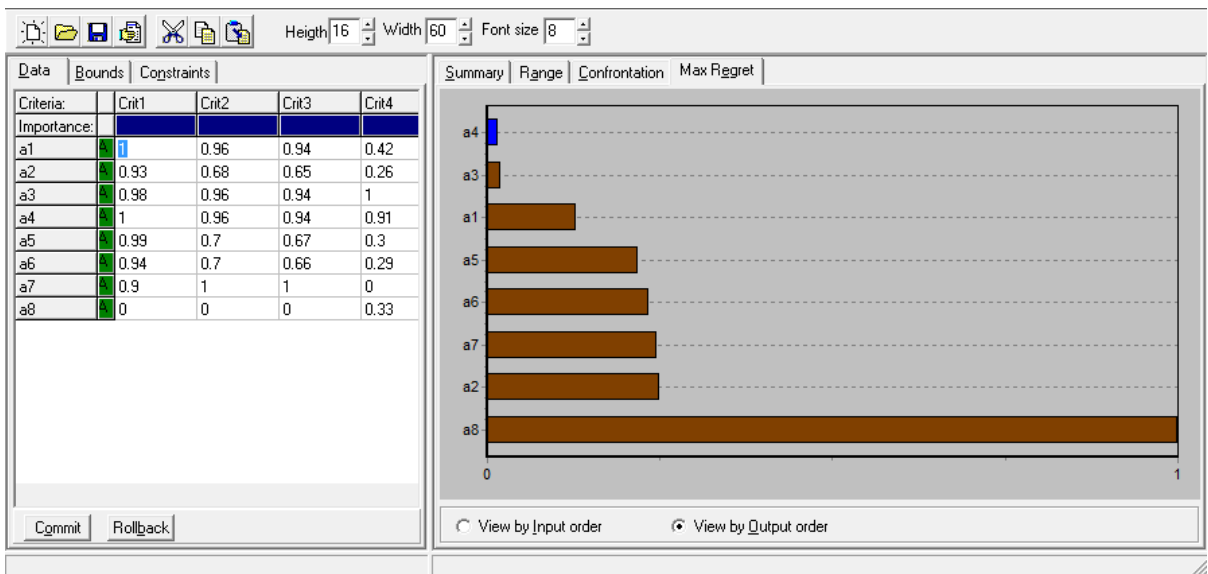

Fig.5 The maximal regrets for $\mathrm{S} 1$.

In a second experiment, $\mathrm{a}_{8}$ was eliminated (a "filtering" procedure), taking into account that this alternative is clearly the worst in terms of all the major evaluation properties used by the VIP analysis, namely "max-regret", "max-min value" and dominance features (note, from fig.4, that it is absolutely dominated by all other alternatives). For the remaining alternatives one could conclude that for $\varepsilon>0.01$ all alternatives, other than $\mathrm{a}_{3}$ and $\mathrm{a}_{4}$, were quasi-dominated by one of these two. Note that the elimination of one or more alternatives from the initial set of solutions, the so called "filtering" procedure, may alter the dominance characteristics of the other alternatives, but this comparison is useful for further exploration of dominance properties of the alternatives which are not eliminated.

In order to analyse, in separate, the relative performance of those two most promising alternatives $\left(a_{3}\right.$ and $a_{4}$, ) with respect to the remaining ones, two more "filtering" experiments were performed, by further elimination of either $a_{3}$ or $a_{4}$ (together with 
$a_{8}$ ). This enabled the conclusion that, in isolation, $a_{3}$ and $a_{4}$ are quasi-optimal with respect to $a_{1}, a_{2}, a_{5}, a_{6}, a_{7}$ for $\varepsilon>0.02$ and $\varepsilon>0.003$, respectively. Finally, a separate comparison of the third more promising solution $\left(a_{1}\right)$, regarding the "min value" and the "max-regret", with $a_{3}$ and $a_{4}$ alone (by filtering all the other alternatives), showed that $\mathrm{a}_{1}$ is quasi-dominated by $\mathrm{a}_{4}$, for $\varepsilon>0.003$ and also by $\mathrm{a}_{3}$ for $\varepsilon>=0.05$. Moreover, $\mathrm{a}_{4}$ quasi-dominates $\mathrm{a}_{3}$ for $\varepsilon=0.02$, that is it becomes quasi-optimal in this particular experiment.

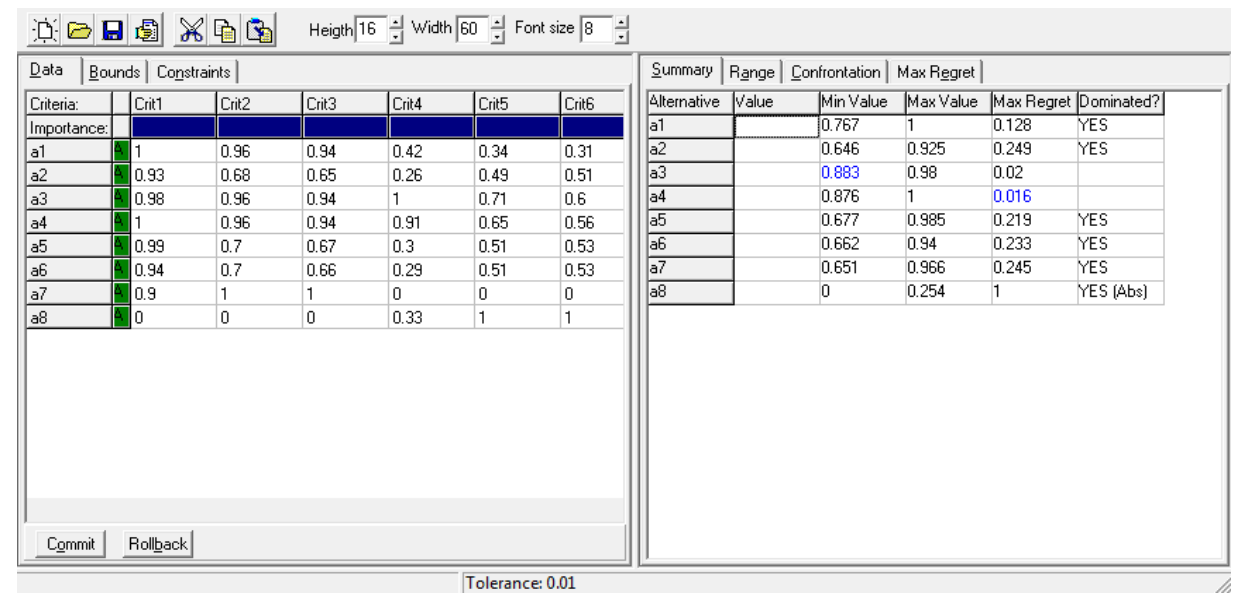

Fig.6 Main features of alternatives, for $\mathrm{S} 1$ and $\varepsilon=0.01$.

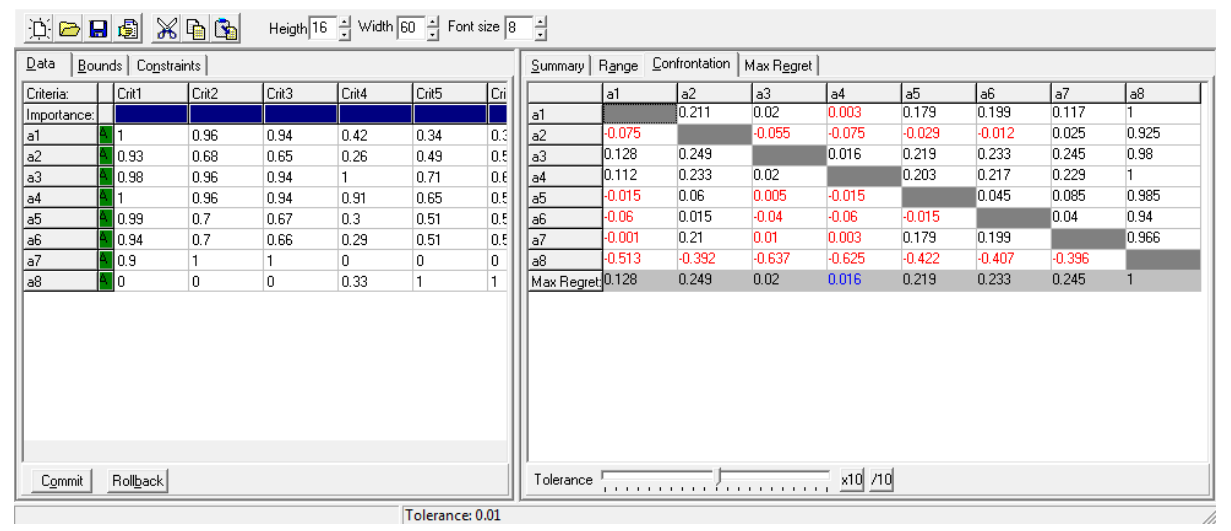

Fig.7 Confrontation table, for $\mathrm{S} 1$ and $\varepsilon=0.01$ 


\section{Second set of experiments (DM2)}

This set of experiments concerns the second decision maker, DM2.

This DM admits that the set of three attributes considered as the more important, under the same basic inequality assumptions as $\mathrm{DM} 1$, for $\mathrm{Br}=0 \%$, correspond to importance parameters (IPs) - namely, $\mathrm{k}_{1}$ (TCB, total carried bandwidth, for $\left.\mathrm{Br} 1=0 \%\right), \mathrm{k}_{4}$ (TRB, total residual bandwidth, for $\mathrm{Br} 1=0 \%$ ), $\mathrm{k}_{7}$ (TAC, total accepted connections, for $\mathrm{Br} 1=0 \%$ ) - the sum of which has, in relative terms, a fixed value, Mip \%, and are all variable. Furthermore, he/she assumes that specific values are assigned to the remaining IPs, $\mathrm{k}_{2}, \mathrm{k}_{3} \mathrm{k}_{5}, \mathrm{k}_{6}, \mathrm{k}_{8}, \mathrm{k}_{9}$, while respecting the above constraints (and of course the normalization equation of the IPs).

So, this DM2, although respecting the same basic inequality relations in S1, concerning the attribute IPs for $\mathrm{Br}=0 \%$, admits that the IPs corresponding to less important attributes, (that is those associated with network conditions with standard blocking probabilities greater than $0 \%$ ) are numerically specified by the DM a anteriori, also leading to a significant simplification of the constraints. This significantly reduces the number of variable IPs and the number of constraints on the IPs. In this case, since six of the IPs are fixed, $k$-optimality regions in the space $\left(\mathrm{k}_{1}, \mathrm{k}_{4}, \mathrm{k}_{7}\right)$, which can be calculated by the VIP software, may be obtained and explored, as referred to in section 2 .

Therefore, the constraints for the constant IPs are now:

$\mathrm{k}_{2}, \mathrm{k}_{3} \mathrm{k}_{5}, \mathrm{k}_{6}, \mathrm{k}_{8}, \mathrm{k}_{9}$ have fixed values such that $\mathrm{k}_{2}+\mathrm{k}_{3}+\mathrm{k}_{5}+\mathrm{k}_{6}+\mathrm{k}_{8}+\mathrm{k}_{9}=1-\mathrm{Mip} / 100$ (10)

and, for the variable IPs:

$$
\begin{array}{ll}
\mathrm{k}_{1}+\mathrm{k}_{4}+\mathrm{k}_{7}=\mathrm{Mip} / 100 & (11 \mathrm{a}) \\
\mathrm{k}_{1}>\mathrm{k}_{4} & (11 \mathrm{~b}) \\
\mathrm{k}_{7}>\mathrm{k}_{4} & (11 \mathrm{c})
\end{array}
$$

In the set of experiments for DM2 we considered Mip=80\% and $\mathrm{k}_{2}=0.054$, $\mathrm{k}_{3}=0.026, \mathrm{k}_{5}=0.02, \mathrm{k}_{6}=0.03, \mathrm{k}_{8}=0.05, \mathrm{k}_{9}=0.02$. This parametrization of $(10),(11)$ corresponds to the constraint set denoted by $\mathrm{S} 2$.

The summary of the main features of the alternatives, in this experiment, is shown in fig. 8, while fig.s 9, 10 show the confrontation table and the max-min range graphics for $\mathrm{S} 2$, respectively. The first major conclusion is that $\mathrm{a}_{2}, \mathrm{a}_{5}, \mathrm{a}_{6}, \mathrm{a}_{7}, \mathrm{a}_{8}$ are absolutely dominated by $a_{4}$ and $a_{3} ; a_{1}$ is dominated by $a_{4}$ but not by $a_{3}$. In this case it is the alternative $a_{3}$ which has, simultaneously, max min value and min max regret, and both $\mathrm{a}_{3}, \mathrm{a}_{4}$ are, in isolation, $\varepsilon$-optimal, for $\varepsilon>0.014$ and $\varepsilon>0.016$, respectively. These alternatives are also k-optimal, that is k-optimality regions, associated with $\mathrm{a}_{3}$ and $\mathrm{a}_{4}$ can be defined in the space $\left(\mathrm{k}_{1}, \mathrm{k}_{4}, \mathrm{k}_{7}\right)$. The execution of the VIP functionality concerning the analysis of the k-optimality properties of $a_{3}$ and $a_{4}$ in the in the space $\left(k_{1}, k_{4}, k_{7}\right)$, enabled to obtain the results in fig. 11 , where the triangle and the trapezium in color represent the projections of the k-optimality regions of $\mathrm{a}_{3}$ and $\mathrm{a}_{4}$, respectively. The table on the right hand-side of the optimality region graphics indicates that the $\mathrm{k}$ optimality region of $\mathrm{a}_{3}$ represents $28.4 \%$ of relative volume in the feasible space $\left(\mathrm{k}_{1}\right.$, $\mathrm{k}_{4}, \mathrm{k}_{7}$ ), while the k-optimality region of $\mathrm{a}_{4}$ represents $71.6 \%$ of that volume. 
Two other sets of experiments, corresponding to variants of the above decision scenario for DM1, where specific proportion relations between some pairs of scaling constants were defined, were carried out. For example instead of $k_{1}>k_{2}$, he/she considers $\mathrm{k}_{1}=\mathrm{b}_{12} \mathrm{k}_{2}$ with a specific value $\mathrm{b}_{12}>1$ and similarly for three other constraints on $\left(\mathrm{k}_{7}, \mathrm{k}_{8}\right),\left(\mathrm{k}_{5}, \mathrm{k}_{4}\right)$ and $\left(\mathrm{k}_{3}, \mathrm{k}_{9}\right)$. That is, four of the inequality constraints in S1 were replaced by proportion relations of the general type (while the other equality/inequality relations still hold):

$$
k_{i}=\beta_{i j} k_{j}
$$

These experiments enabled this DM to fix, in quantitative terms, possible relative importance values, between certain pairs of attributes and evaluate the effect of such choices. The results of these experiments are not presented here since they were not significantly different, in qualitative terms, from the base scenario for DM1, namely that five alternatives are dominated by $\mathrm{a}_{3}$ or $\mathrm{a}_{4}$, four being absolutely dominated, and $\mathrm{a}_{3}$ (with max-min value) and $\mathrm{a}_{4}$ (with min-max regret) are still the most promising solutions. Details of these results can be seen in a short report [Craveirinha et al. 2018].

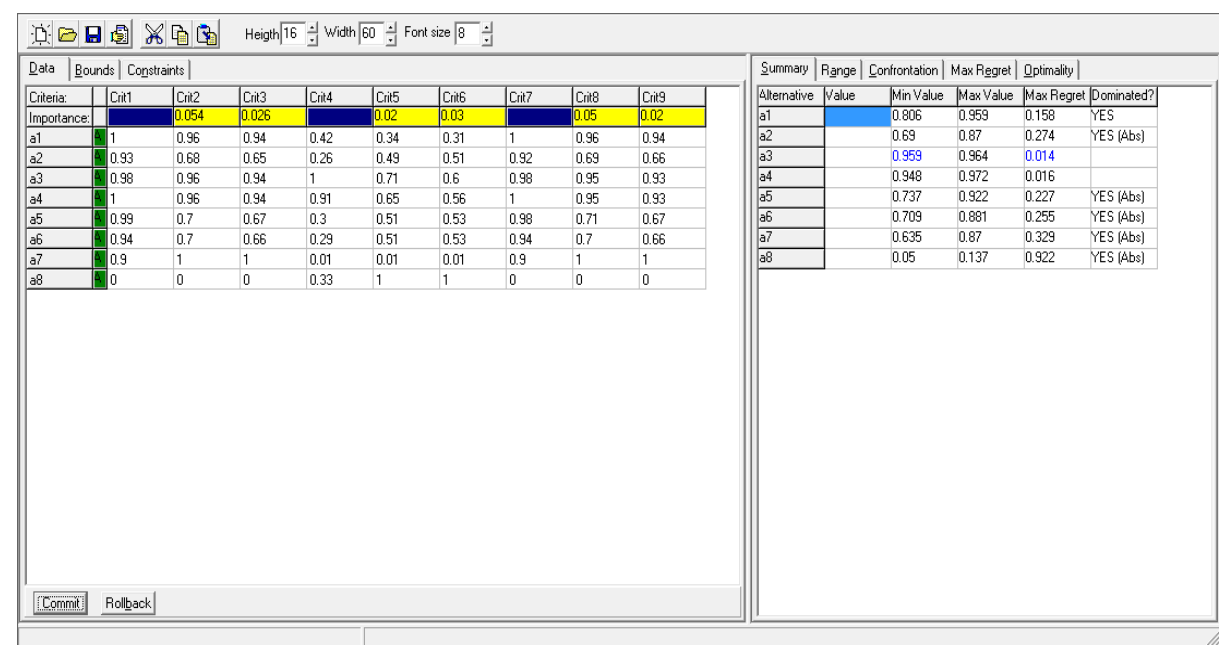

Fig. 8 Main features of alternatives, for S2 
J. Clímaco, J. Craveirinha, and L. Martins 21

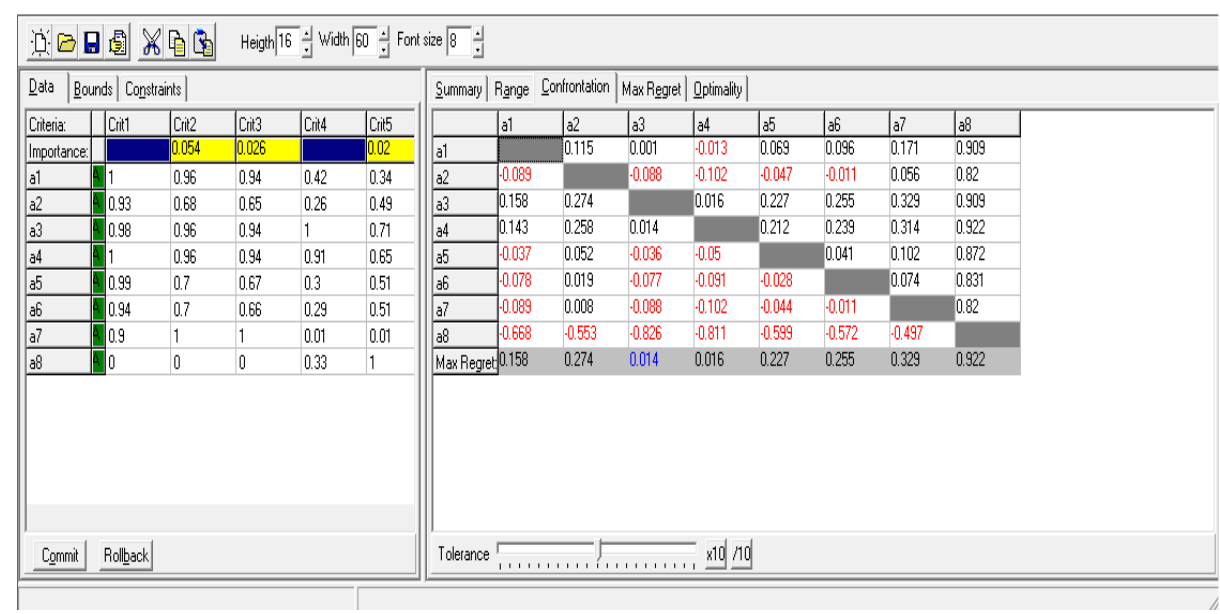

Fig. 9 Confrontation table, for S2

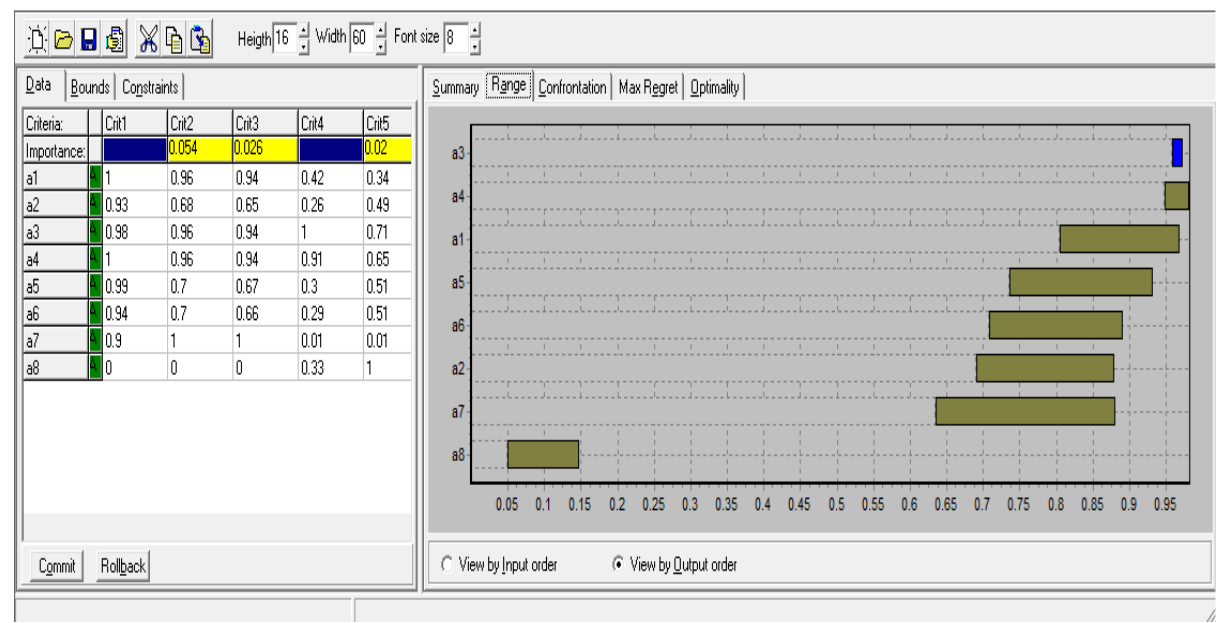

Fig. 10 Min-max ranges for S2 


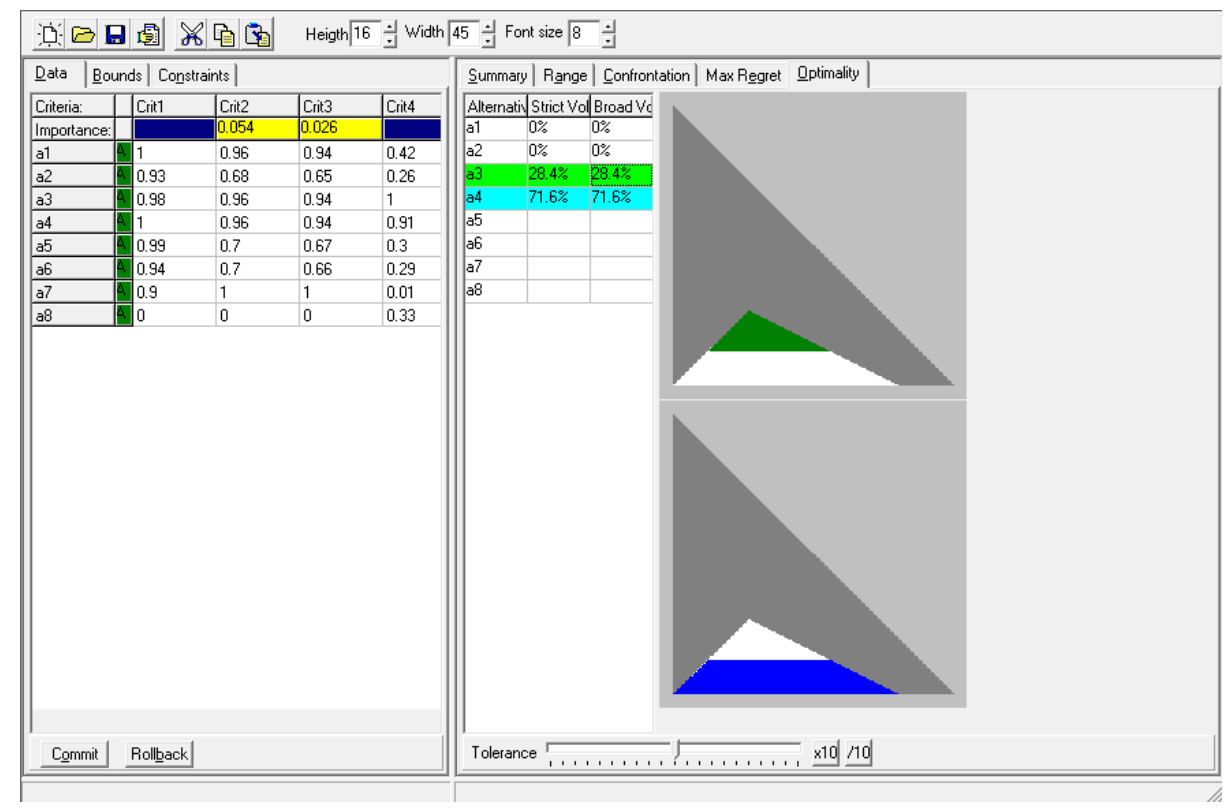

Fig. 11 k-optimality regions of $\mathrm{a}_{3}$ and $\mathrm{a}_{4}$ for $\mathrm{S} 2$. The shaded areas represent projections of volumes in the space $\left(\mathrm{k}_{1}, \mathrm{k}_{4}, \mathrm{k}_{7}\right)$, onto a two dimensional space.

\section{Synopsis of results with one DM}

The two sets of separate experiments, for DM1 and DM2, showed two variants of decision scenarios, considering the same basic underlying assumptions regarding the relative importance of the criteria, as assigned, by typical network designers, to TCB, TAC and TRB network performance measures. Two other sets of experiments, in this same framework of assumptions, considering one DM, were carried out, as mentioned above (see details in the research report [Craveirinha et al.2018]).

The major conclusions, from all these experiments, were:

i) the routing method $\left(a_{8}\right)$ using minimal hop paths is absolutely dominated by all the other methods, so it is consistently a very poor solution to be avoided, thence confirming, in a systematic and mathematically consistent manner (in a scenario of incremental traffic) all previous empirical results in the telecommunication literature concerning the evaluation of this routing method;

ii) the two 'best' alternatives were the bi-criteria routing methods, using either the Euclidian or the Chebyschev distances (both using the normalization coefficients WA) to the ideal optimum in terms of load cost and hop count, namely $a_{3}$ and $a_{4}$. 
iii) these two more promising alternatives, $a_{3}$ and $a_{4}$ either dominated, absolutely dominated or quasi-dominated the other routing methods, with slight variations of these features in the different scenarios, therefore they are quasi-optimal vis a vis the other six remaining methods;

iii) the next method with reasonable behaviour in terms of max-min value and maximal regret, in all scenarios, was the bi-criteria method $a_{1}$, which uses the normalization coefficients WA and priority regions; nevertheless, this alternative is quasidominated by $\mathrm{a}_{3}$ and by $\mathrm{a}_{4}$ for relatively low values of $\varepsilon$, in all these scenarios.

Furthermore, one can say that $\mathrm{a}_{4}$ is the one alternative which has, overall, stronger dominance properties in these sets of experiments. In fact, $a_{4}$ quasi-dominates $a_{3}$ except for DM2, a case in which $\mathrm{a}_{3}$ has the advantage of being the alternative with max min value and min max regret (although at a very short distance from $a_{4}$ in this respect), but $\mathrm{a}_{4}$ has a significantly better k-optimality feature, namely a k-optimality region with a relative volume in the feasible space $\left(\mathrm{k}_{1}, \mathrm{k}_{4}, \mathrm{k}_{7}\right)$, more than 2,5 times greater than the corresponding volume for $a_{3}$. One may conclude that, although $a_{3}$ should not be disregarded as routing method with very good overall network performance, overall, in this application context, $\mathrm{a}_{4}$ would be the selected bi-criteria routing method.

\subsection{Study for cooperative group decision}

Next we considered the extension of the experimental study to the case of face-toface cooperative group decision with a facilitator. Although some general assumptions on relative importance of the network performance metrics may be common to most network designers, the specification of relations between the criteria IPs may, in some few cases, vary significantly from one DM to another. Furthermore, as noted above, this may be a realistic decision setting in the context of a major network operator, since more than one engineer/network designer is often involved in a decision which has decisive impact in terms of network performance and cost. As stressed above, the group decision support model, based on the VIP software (see [Dias \& Clímaco 2005]), was designed in order to reflect to each DM the consequences of the other DMs inputs. Hence, each DM is confronted with analogous images of the decision group elements' inputs. However, in the addressed decision problem, a routing method has to be chosen. Two situations may occur in the MA analysis process: either one alternative becomes accepted by all the DMs, as the more favourable, or two or more alternatives should be considered, in equal standing, by the DMs. In the latter case, the facilitator will be the 'head of the team', i.e. the DM who will be accountable to the operator management for the implementation of the routing solution, whom we also may designate as 'last resort DM'. In this case, he/she will have to make a final choice of a method among a final short list of alternatives, as suggested in the short paper [Clímaco et al. 2015].

Hereafter, we consider a cooperative group decision setting comprising the decision makers DM1, DM2 whose system of preferences concerning the relations between IPs, was described in the previous section, plus a third decision maker, DM3, who has some distinct qualitative feature, concerning the relative importance of major 
performance criteria. This DM3, is, in a sense, out of the 'main stream', in terms of common preferences, vis a vis the other DMs, namely by considering that some of the inequality relations between IPs, assumed by DM1 and DM2, should be reversed. Namely, DM3 considers that TRB (Total Residual Bandwidth) is more important than TCB (Total Carried Bandwidth) and TAC (Total Accepted Connections), for the same level of blocking probability, that is he/she favours more short term minimisation of the usage of networks resources than total mean carried bandwidth or mean total accepted connections. This type of preferences may favour other types of routing solutions as compared with the ones favoured by the analysis of DM1 and DM2.

The new constraints for the IPs are:

$$
\begin{gathered}
K_{(i) T C B} \prec K_{(i) T B R}(i=1,2,3) \\
K_{(i) T A C} \prec K_{(i) T B R} \quad(i=1,2,3)
\end{gathered}
$$

These constraints replace (c4) and (c5) in the constraint set S1, the remaining constraints of which remain unchanged. The new constraint set, characterizing DM3, will be denoted by S3. The major results of the analysis of alternatives by this DM are shown in the figures B1 (summary of results), B2 (confrontation table) and B3 (maxmin ranges) of Appendix B.

The main results from this analysis are:

i) Six alternatives $\left(a_{1}, a_{2}, a_{4}, a_{5}, a_{6}, a_{7}\right)$ are dominated by $a_{3}$ and five $\left(a_{1}, a_{2}, a_{5}, a_{6}, a_{7}\right)$ are dominated by $\mathrm{a}_{4}$;

ii) Alternative $a_{3}$ has 'max-min value' and 'min max regret' and dominates $a_{4}$, this last feature being in contrast with the analysis of DM1 and DM2, but still $a_{4}$ is the second more favorable in terms of 'max-min value' and 'max-min regret';

iii) The only alternative not dominated by $a_{3}$ is $a_{8}$, but $a_{8}$ still has very poor performance in terms of 'min value' and 'max regret'.

Therefore, the major conclusion is that, for DM $3, a_{3}$ is overall the best compromise alternative, since it dominates the second more favourable, $\mathrm{a}_{4}$.

Remember that we are assuming a cooperative group decision analysis environment, where the three DMs are confronted with the preference choices and results of the other decision group elements' inputs. Subsequently, we now address the interplay between the tolerance $\varepsilon$, defining quasi-dominance relations between two alternatives, and $\alpha$-majority relations, which may be analysed according to the concepts in [Dias \& Clímaco 2005], highlighted in section 2. For this purpose, exemplifying in our case study, we will consider the aggregation of preferences at the output level and identify two major conclusions concerning $\varepsilon$ dominance properties of $a_{3}, a_{4}$, with respect to five alternatives $\left(a_{2}, a_{5}, a_{6}, a_{7}, a_{8}\right)$. This assumes that, after a preliminary discussion, all DMs agreed that the two more promising alternatives are $\mathrm{a}_{3}$ and $\mathrm{a}_{4}$, consistently with the results previously presented for the three DMs. Let us apply an extension of the quasi-dominance concept to multiple DMs. Given DMd (i.e. a DM specified by "d"), characterized by a set $\mathrm{T}_{\mathrm{d}}$ of admissible importance parameter values, we denote the results/propositions, assuming an $\alpha$ majority rule: " $a_{\mathrm{i}}$ quasi-dominates $\mathrm{a}_{\mathrm{j} 1,}, \mathrm{a}_{\mathrm{j} 2}, \ldots, \mathrm{a}_{\mathrm{jm}}$ " with tolerance $\varepsilon$, for a majority of $\alpha$ decision makers" by:

$$
\mathrm{a}_{\mathrm{i}} \Delta_{\varepsilon(\alpha)} \mathrm{a}_{\mathrm{j} 1}, \mathrm{a}_{\mathrm{j} 2}, \ldots, \mathrm{a}_{\mathrm{jm}}
$$


We considered this type of proposition in the context of our experiment, in order to examine and compare the quasi-dominance properties of $\mathrm{a}_{3}$ and $\mathrm{a}_{4}$ with respect to $\mathrm{a}_{2}$, $\mathrm{a}_{5}, \mathrm{a}_{6} \mathrm{a}_{7}, \mathrm{a}_{8}$, in terms of the $\alpha$ majority rule. These results can be represented by the graphics of fig. 12 .

For example, in fig. 12a, we have:

$$
a_{3} \Delta_{0.01(2 / 3))}\left(a_{2}, a_{5}, a_{6}, a_{7}, a_{8}\right)
$$

This means that $a_{3}$ dominates $a_{2}, a_{5}, a_{6} a_{7}, a_{8}$ for two of the three DMs, in our case study, with a tolerance of 0.01 . It also holds, in fig. 12a:

$$
\mathrm{a}_{3} \Delta_{0.4(1)}\left(\mathrm{a}_{2}, \mathrm{a}_{5}, \mathrm{a}_{6}, \mathrm{a}_{7}, \mathrm{a}_{8}\right)
$$

This means that $\mathrm{a}_{3}$ dominates $\mathrm{a}_{2}, \mathrm{a}_{5}, \mathrm{a}_{6} \mathrm{a}_{7}, \mathrm{a}_{8}$ for all the three DMs, in our case study, with a tolerance of 0.4 .

Similar information can be seen in the graphic of fig. 12.b, in this case concerning the dominance properties of $a_{4}$ with respect to $a_{2}, a_{5}, a_{6} a_{7}, a_{8}$.

We can conclude that the interplay between the tolerance $\varepsilon$, defining quasidominance relations, and $\alpha$-majority relations, suggests that, overall, $\mathrm{a}_{4}$ has somehow better $\varepsilon$-dominance properties than $\mathrm{a}_{3}$ for a $2 / 3$ majority of DMs. Moreover, as seen in the previous experiments, the $\varepsilon$-optimality properties of these two alternatives (when analysed separately in confrontation with the other alternatives) for DM1 and DM2 and the k-optimality properties for DM2, discussed above, also suggest that this conclusion is defensible for our group decision process, although we can say that $\mathrm{a}_{3}$ is still a very good routing alternative. If we considered the third more favourable alternative, $\mathrm{a}_{l}$, as an additional argument in the properties of type (14) above, we might further conclude that, for even stronger reasons, $\mathrm{a}_{4}$ outperforms $\mathrm{a}_{3}$ in terms of a $\varepsilon-2 / 3$ majority rule.
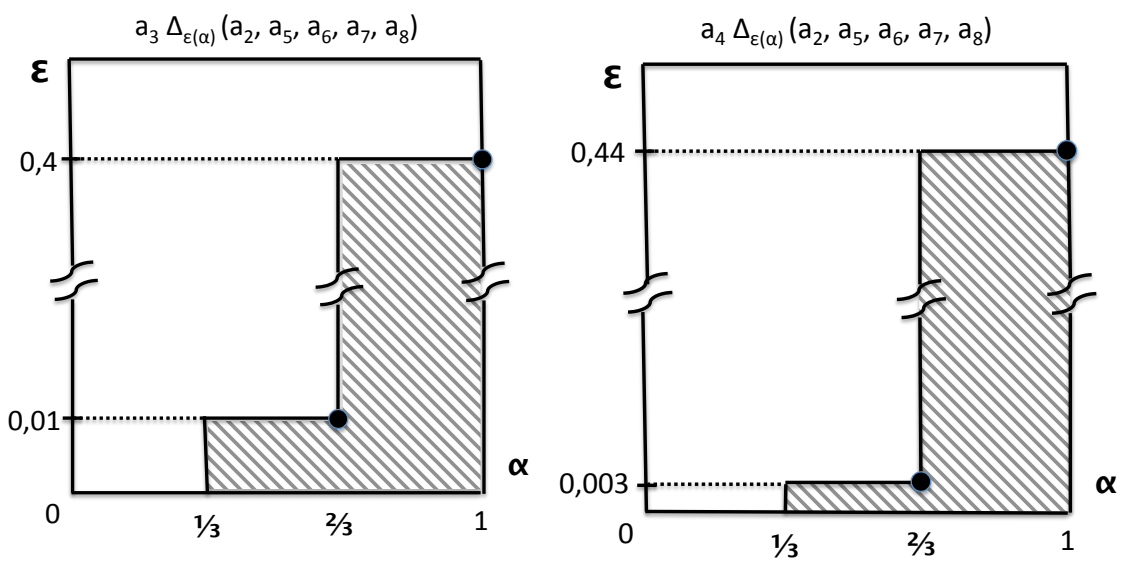

Fig. 12a- $\varepsilon(\alpha)$-majority relations for $a_{3}$ Fig. $12 \mathrm{~b}-\varepsilon(\alpha)$-majority relations for $a_{4}$ 


\section{Conclusions and Further Work}

After analyzing the reasons why it is necessary to evaluate the performance of flow oriented routing models through relevant global network performance measures, we concluded that the comparison and ultimately the choice - by a network design expert - of "a routing method with better overall performance" in a given network context, may easily become a difficult task, involving what is, in fact, a complex decision problem. Such decision problem should, in our view, be tackled with methodologically sound OR-decision support techniques, in the framework of multi-attribute decision analysis.

A major conclusion of our study was to show the practical usefulness and great potential, from a methodological point of view, of using a multi-attribute analysis model, dealing with incomplete information, in this network design decision process. This has to do with the fact that flow oriented routing models, due to their inherent limitations, have to be evaluated through global network performance parameters, corresponding to the attributes of our decision problem, that are often conflicting and incommensurate. Moreover, the incomplete and imprecise information features of the proposed MA model stems from the fact that the scaling constants (or importance parameters), associated with the considered attributes are not fixed a priori, although various inequality, proportion relations, or specific values for some of these IPs, can be set a priori by the decision maker(s), assuming possible different scenarios for such relations. We have specified a multi-attribute (MA) analysis model, based on the VIP Analysis software (which considers variable interdependent importance parameters), for comparing and selecting routing methods, in terms of multiple network performance measures, enabling the consideration by the network designer (decision maker) of multiple scenarios concerning different forms of valuation of the relative importance of the network performance measures. Furthermore, we considered the application of the extension of this decision analysis model, based on the VIP decision support tool, for dealing with this problem, in the case of face-to-face cooperative group decision.

The application of this model to a case study involving the comparison and selection of one of various flow-oriented routing methods, based on bi-criterion shortest paths and single-criterion shortest paths algorithms (using as path metrics load cost and hop count) in the context of transport networks with incremental traffic, showed, as second important conclusion, the total adequacy of this VIP based multi-attribute analysis model, that assumes an additive value function under imprecise information, to tackle the formulated decision problem. Furthermore, in this type of study, we could make the most of this learning oriented interactive tool.

Moreover, from a network design point of view, a number of relevant conclusions could be drawn from our extensive experimental case study, both in the case of a single decision maker and in the case of three cooperative decision makers. In the case study we considered nine attributes associated with total carried bandwidth, total residual bandwidth and total number of accepted connections, in different load/overload conditions, in a transport network with incremental traffic. Firstly, the routing method $\left(a_{1}\right)$ using minimal hop paths is absolutely dominated by all the other methods, so it is consistently a very poor solution to be avoided, thence confirming, in 
a systematic and mathematically consistent manner all previous experimental results in the literature, concerning the evaluation of this routing method. Secondly, the two "best" routing procedures, were two specific bi-criteria routing methods, with a certain set of normalization coefficients depending on the network conditions, which either dominated, absolutely dominated or quasi-dominated the other routing methods. Thirdly, taking into account that one of these two had the strongest dominance properties, we may conclude that - although the other bi-criterion method should not be disregarded as routing method with very good overall network performance - the recommended bi-criteria routing method, in this particular network and decision environment, was the bi-criteria routing method which used a Chebyschev distance to the ideal optimum. This conclusion was still valid, in a cooperative group decision environment with three decision makers, in particular, by applying a $2 / 3$ majority rule, following the concepts in [Dias \& Clímaco 2005], and also taking into account the quasi dominance and k-optimality features of the two most promising solutions.

Further work on this research line might involve the application of the developed MA model to other type of network environments and to other problems of telecommunication network design.

Acknowledgments. Acknowledgement: This work was financially supported by FEDER Funds and National Funds through FCT - 'Fundação para a Ciência e a Tecnologia' under the projects UID/MULTI/00308/2019 and CENTRO-01-0145FEDER-029312.

\section{Appendix A - Variants of the bi-criteria routing model}

Concerning the bi-criteria routing model, in two of the considered variants, $a_{1}, a_{2}$, the aggregation of the bi-criteria preferences was performed by using preference regions in the objective function space. These regions were obtained by defining required and acceptable values $\mathrm{m}_{\text {req }}^{\mathrm{i}}, \mathrm{m}_{\mathrm{ac}}^{\mathrm{i}}(\mathrm{i}=1,2)$, specified by the coordinates corresponding to points at distances, taken from the optimal point coordinates, at $1 / 3$ and $2 / 3$ of the variation range of the corresponding function $\mathrm{m}^{\mathrm{i}}\left(\mathrm{r}_{\mathrm{s}}\right)$, as shown below ( $c f$. eq. A3). In these variants, the non-dominated paths were obtained by calculating kshortest paths, using the additive path cost function (A1), and choosing the first solution in the highest non-empty priority region:

$$
\min _{r_{s}} m\left(r_{s}\right)=\sum_{l_{k} \in r_{s}}\left(m_{k}^{* 1}+m_{k}^{* 2}\right)
$$

where $m_{k}^{* i}=\varepsilon_{i} m_{k}^{i}$ is the normalized value of the cost function $\mathrm{m}^{\mathrm{i}}$ at arc $l_{k},(\mathrm{i}=1,2)$. The normalizing coefficients $\varepsilon_{\mathrm{i}}$ (such that $\varepsilon_{1}+\varepsilon_{2}=1$ ) were calculated in two different forms, in the various variants of the model. Let $\mathrm{p}^{\mathrm{i}}$ de the minimal value of the two path metrics $\mathrm{m}^{\mathrm{i}}\left(\mathrm{r}_{\mathrm{s}}\right) \quad(\mathrm{i}=1,2)$, and $\Delta^{i}$ the range of values of $\mathrm{m}^{\mathrm{i}}\left(\mathrm{r}_{\mathrm{s}}\right)$ defined in terms of the Nadir point $\left(\mathrm{M}^{1}, \mathrm{M}^{2}\right)$, in the objective function space:

$$
\Delta^{i}=M^{i}-o p^{i} ; M^{i}=m^{i}\left(\arg \left\{\min m^{j}\left(r_{s}\right)\right\}\right)(\mathrm{i}=1,2) \wedge \mathrm{j} \neq \mathrm{i}
$$


Therefore, the required and acceptable values $\mathrm{m}_{\text {req }}^{\mathrm{i}}, \mathrm{m}_{\mathrm{ac}}^{\mathrm{i}}(\mathrm{i}=1,2)$ were calculated as follows:

$$
m_{a c}^{i}=o p^{i}+\frac{2}{3} \Delta^{i} ; \quad m_{r e q}^{i}=o p^{i}+\frac{1}{3} \Delta^{i}
$$

Concerning the first set of normalizing coefficients, WA, it is obtained by equalizing the two ranges $\varepsilon_{i} \Delta_{i}(i=1,2)$, leading to:

$$
\varepsilon_{i}=\frac{1}{\Delta^{i}}\left(\sum_{k=1}^{2} \frac{1}{\Delta^{k}}\right)^{-1} i=(1,2) \quad(\mathrm{A} 4)
$$

Note that these coefficients are calculated each time a VC (node to node virtual connection) is established.

The second set of normalizing coefficients, WB, is calculated (cf. [Martins et al. 2013]) by considering the average of each path metric, for the current state of the network links $1_{\mathrm{k}}$ :

$$
\overline{m_{k}^{i}}=\frac{\sum_{l_{k} \in L} m_{k}^{i}}{|L|}
$$

where $\mathrm{L}$ is the set of network links, $|L|$ denotes the cardinal of $\mathrm{L}$ and $\mathrm{m}_{\mathrm{k}}{ }^{\mathrm{i}}$ is the cost associated with metric $\mathrm{m}^{\mathrm{i}}$, considering all current occupations in link $1_{\mathrm{k}}$. The equalization of the variation ranges, considering these averages $\overline{m_{k}^{i}}$ leads to:

$$
\varepsilon_{1}=\frac{1}{1+\overline{m_{k}^{1}}} \quad \varepsilon_{2}=1-\varepsilon_{1}
$$

Note that, in this case, the coefficients don't have to be calculated for each VC since they depend on the average metric values.

The other variants of the routing model seek non-dominated solutions which minimize either the Euclidian or the Chebyschev distance to the ideal optimum, also considering the two different sets of normalizing coefficients WA, WB, determined as explained above. This leads two four variants of the routing model, $\mathrm{a}_{3}, \mathrm{a}_{4}, \mathrm{a}_{5}, \mathrm{a}_{6}$. Further details can be seen in [Martins et al. 2013]. 


\section{Appendix B - Results for decision maker DM3}

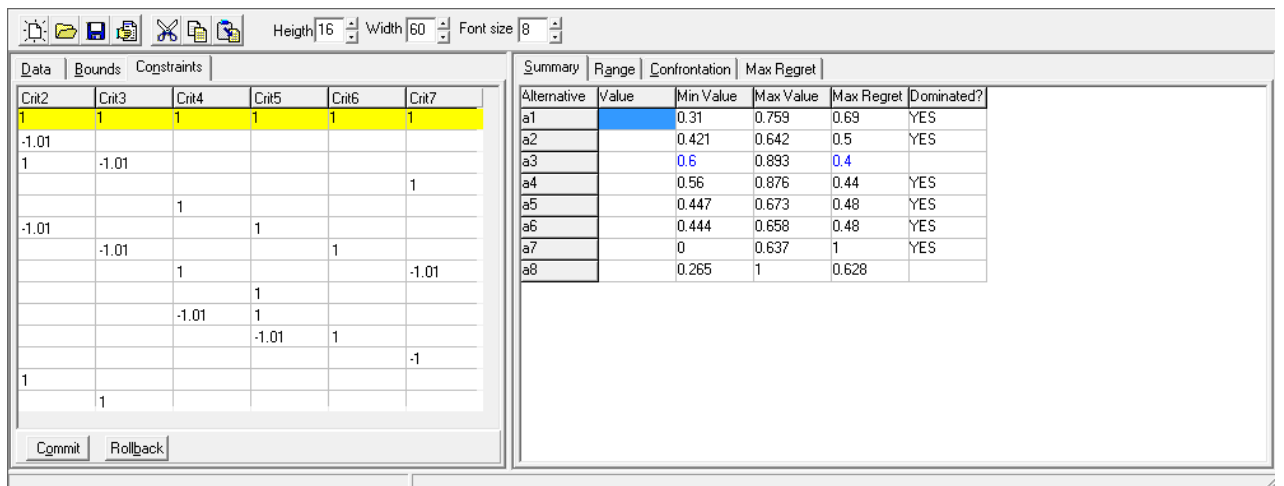

Fig. B1 Summary of results for S3

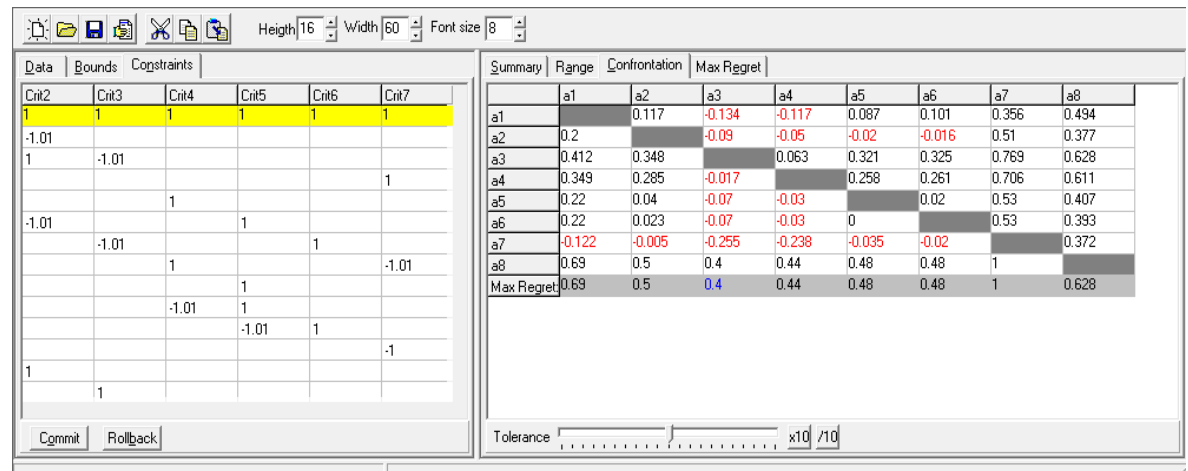

Fig. B2 Confrontation table for S3

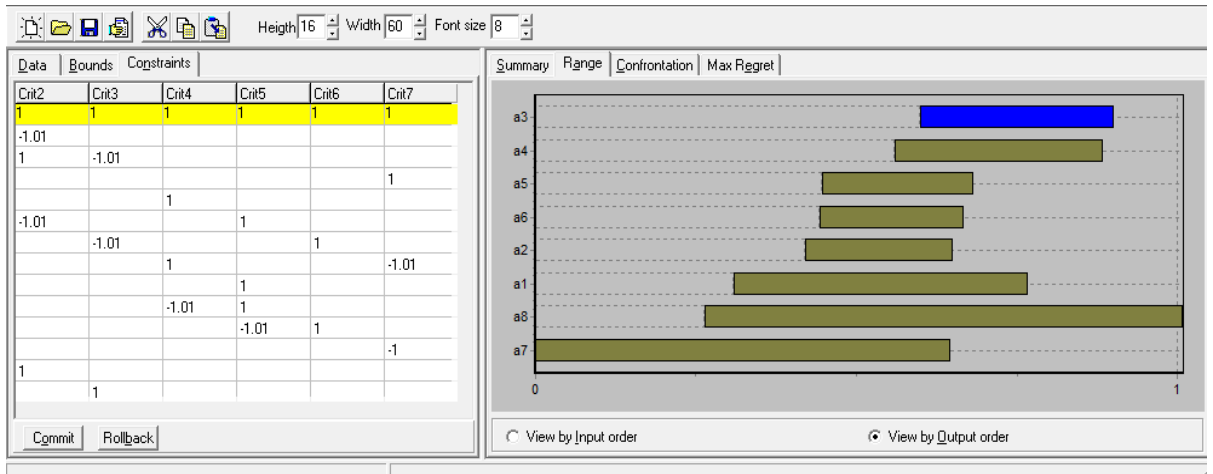

Fig. B3 Min-max ranges for S3 
Acknowledgement: This work was financially supported by FEDER Funds and National Funds through FCT - 'Fundação para a Ciência e a Tecnologia' under the projects UID/MULTI/00308/2013 and CENTRO-01-0145-FEDER-029312.

\section{References}

Clímaco, J. and Craveirinha, J. (2005). Multicriteria analysis in telelecommunications design and planning- problems and issues. Chapt. 22 in "Multiple Criteria Decision Analysis: State of the Art Surveys"”, pp. 899-951, eds. F. Figueira, S. Greco, and M. Erghot, Int. Series in Operations Research and Management Science, SpringerMedia, New York.

Clímaco, J., Craveirinha, J., Girão-Silva, R. (2016). Multicriteria analysis in telecommunication network planning and design: A survey. In S. Greco, M. Ehrgott, R.J. Figueira (eds.) "Multiple Criteria Decision Analysis: State of the Art Surveys", International Series in Operations Research \& Management Science, vol. 233, pp. 11671233. Springer New York.

Clímaco, J., Craveirinha, J., Pascoal, M. (2007). Multicriteria routing models in telecommunication networks - overview and a case study. Chapt. 1 in Y. Shi, D. Olson, A. Stam (eds.) "Advances in multiple criteria decision making and human systems management: knowledge and wisdom", book edited in honor of Milan Zeleny, pp. 17-46, IOS Press.

Clímaco, J. and Martins, E.Q. (1982). A bicriterion shortest path algorithm. Eur.J. Operational Research (11), pp. 399-404.

Craveirinha, J., Girão-Silva, R., Clímaco, J. (2008). A meta-model for multiobjective routing in MPLS networks. Central European Journal of Operations Research 16(1), pp. 79-105.

Clímaco, J., Craveirinha, J., Martins, L. (2015). Cooperative Group Multi-attribute Analysis of Routing Models for Telecommunication Network. Proc. GDN 2015, Conf. Group Decision and Negotiation 2015, pp. 177-184, 22-26 June, Warsaw, Poland, Ed. by B. Kaminski, G. Kersten, P. Szufel, M. Jakubczyk, T. Wachowicz, Warsaw School of Economics Press.

Craveirinha, J., Clímaco, J., Martins, L. (2018). Additional computational experiments on a comparative study of the performance of flow-oriented routing methods in telecommunication networks. Research Report, INESC Coimbra RR (to appear).

Dias, L.C. and Clímaco, J.N. (2000). Additive aggregation with variable interdependent parameters: The VIP analysis software. The Journal of the Operational Research Society 51(9), pp. 1070-1082.

Dias, L.C. and Clímaco, J. N. (2005). Dealing with imprecise information in group multicriteria decisions: a methodology and a GDSS architecture. European Journal of Operational Research 160(2), pp. $291-307$.

Fortz, B. and Thorup, M. (2002). Optimizing OSPF/IS IS weights in a changing world. IEEE Journal on Selected Areas in Communications, 20(4), pp. 756 - 767.

MEF 10.3 (2013). Ethernet Services Attributes Phase 3.

Niven-Jenkins, B., Brungard, D., Betts, M., Sprecher, N., Ueno, S. (2009). Requirements of an MPLS Transport Profile. RFC 5654, September. 
Iyer, G., Agrawal, P., Cardozo, R. S. (2013). Performance comparison of Routing Protocols over Smart Utility Networks: A Simulation Study. Proc. Globecom 2013 Workshop - The 5th IEEE International Workshop on Management of Emerging Networks and Services, pp. 969-973.

Keeney, R.L. and Raiffa, H. (1976). Decisions with multiple objectives: preferences and value trade- offs. Wiley.

Kuipers, F., Korkmaz, T., Krunz, M., Mieghem, P. (2002a). An overview of constraint-based path selection algorithms for QoS routing. IEEE Communications Magazine 40(12), pp. 50-55.

Kuipers, F., Korkmaz, T., Krunz, M., Mieghem, P. (2002b): A review of constraint-based routing algorithms. Tech. rep., Delft University of Technology.

Martins, L., Lopes, J., Craveirinha, J., Clímaco, J., Cadime, R., Monica, C. (2013). Network performance improvement through evaluation of bicriteria routing methods in transport networks. In: Network and Service Management (CNSM), 2013 Proc. 9th International Conference on Network and Service Management, pp. 34-41.

Medhi, D. and Ramasamy, K. (2018). Network Routing: Algorithms, Protocols, and Architectures (Second Edition). In The Morgan Kaufmann Series in Networking, Boston, USA.

Meghanathan, N. and Milton, L. C. (2009). A Simulation Based Performance Comparison Study of Stability-Based Routing, Power-Aware Routing and LoadBalancing On-Demand Routing Protocols for Mobile Ad hoc Networks. Proc. 2009 Sixth International Conference on Wireless On-Demand Network Systems and Services, Snowbird, UT, pp. 3-10.

Mitra, D., Morrison, J.A., Ramakrishnan, K.G. (1999). Optimization and design of network routing using refined asymptotic approximations. Performance Evaluation An International Journal 36-37, pp. 267-288.

Orlowski, S., Wessaly, R., Piíoro, M., Tomaszewski, A. (2010). SNDlib 1.0survivable network design library. Networks 55(3), pp. 276-286.

Pióro, M. and Medhi, D. (2004). Routing, Flow, and Capacity Design in Communication and Computer Networks. Morgan Kaufmann Publishers Inc., San Francisco, CA, USA.

Sllame, A. M. , Raey, A., Mohamed, B., Alagel, A. (2015). Performance Comparison of VoIP over Wireless Ad Hoc Networks Using Different Routing Protocols and Queuing Techniques. Proc. International Symposium on Networks, Computers and Communications (ISNCC), pp. 1-6, Hammamet. 